\title{
GOAL RELEVANCE INFLUENCES PERFORMANCE MONITORING AT THE LEVEL OF THE FRN AND P3 COMPONENTS
}

Wioleta Walentowska (1,2), Agnes Moors (2,3), Katharina Paul (2), and Gilles Pourtois (2)

(1) Psychophysiology Laboratory, Institute of Psychology, Jagiellonian University, Kraków, Poland

(2) Department of Experimental Clinical and Health Psychology, Ghent University, Belgium

(3) Research Group of Quantitative Psychology and Individual Differences, Center for Social and Cultural Psychology, KU Leuven, Belgium

Running head: Goal relevance influences the FRN component

Corresponding address:

Wioleta Walentowska

Psychophysiology Laboratory

Jagiellonian University

Ingardena 6

30-060 Kraków

Poland

e-mail address: wioleta.walentowska@uj.edu.pl

phone number: +48 126632408 
Abstract

The Feedback-Related Negativity (FRN) provides a reliable ERP marker of performance monitoring (PM). It is usually larger for negative compared to positive feedback, and for unexpected relative to expected feedback. In two experiments, we assessed whether these effects could be modulated by goal relevance, defined as feedback informativeness (reliability) and/or impact on a person's goals. 64channels EEG was recorded while 30 participants (in each experiment) performed a speeded Go/NoGo task across blocks in which the feedback on task performance was deemed either relevant or not. At the ERP level, the FRN component was larger for (frequent) negative compared to (deviant) positive feedback exclusively when the feedback was relevant (Experiment 1). When the probability of positive and negative feedback was balanced (Experiment 2), this valence-driven FRN effect was absent. However, across these two experiments, the FRN was always larger for irrelevant than relevant feedback. Moreover, the subsequent P300 component was larger for feedback in the relevant than the irrelevant blocks. This effect was valence-unspecific in Experiment 1, while in Experiment 2 larger P3 amplitudes were recorded for negative than positive (relevant) feedback. Across the two experiments, a larger Correct-Related Negativity (CRN) in the irrelevant than relevant context was also observed, suggesting that PM is flexible. These ERP findings indicate that goal relevance influences feedback (and response) processing during PM, with two non-overlapping neurophysiological effects: It gates reward prediction error brain mechanisms (FRN effect), before enhancing subsequent motivational processes (P300 effect).

Keywords: ERP; FRN; P3; CRN; Performance Monitoring; Goal Relevance 


\section{Introduction}

Performance monitoring (PM) is based on the processing of both internal and external cues or signals. When internal (i.e., motor-based) evidence is not available or has not accumulated properly, processing of external (feedback-related) incentives usually prevails and guides the course of PM. A large number of neurophysiological studies and models have already shown that the Feedback-Related Negativity (FRN) reflects external feedback information processing during PM, and more specifically, reward prediction error (Holroyd \& Coles, 2002; Holroyd, Pakzad-Vaezi, \& Krigolson, 2008; Walsh \& Anderson, 2012). When the feedback on task performance informs about a mismatch between the expected and/or desired outcome and the actual outcome (usually a response error or incorrect decision preceding feedback onset), a phasic and negative-going wave is elicited around $250-300 \mathrm{~ms}$ after its onset over fronto-central locations along the midline. Consistent with the reward prediction error account, the amplitude of the FRN is larger for monetary losses compared to wins (Gehring \& Willoughby, 2002), for negative compared to positive symbolic performance feedback (Miltner, Braun, \& Coles, 1997; Nieuwenhuis, Holroyd, Mol, \& Coles, 2004), and for unexpected relative to expected events (Hajcak, Moser, Holroyd, \& Simons 2007; Pfabigan Alexopoulos, Bauer, Lamm, \& Sailer, 2011; von Borries, Verkes, Bulten, Cools, \& de Bruijn, 2013). However, the exact functional meaning of the FRN component, and by extension, the cognitive process that it reflects, remains debated in the literature (Proudfit, 2015; Ullsperger, Fischer, Nigbur, \& Endrass, 2014b). As outlined above, while some studies emphasized the role of the FRN in processing the (negative) valence of performance feedback irrespective of their likelihood (Hajcak, Moser, Holroyd, \& Simons, 2006), other studies have reported evidence for the opposite statement (i.e., the FRN is sensitive to expectedness, and more specifically, unexpected events, regardless of their valence; see Ferdinand, Mecklinger, Kray, \& Gehring, 2012). In this study, we test the prediction that neither valence nor expectedness provides the critical dimension to explain FRN amplitude variations during PM, but rather 'goal relevance' (see also Gentsch, Grandjean, \& Scherer, 2013).

The concept of goal relevance plays a central role in research on emotions (Frijda, 1986), attention (Folk \& Remington, 2008), and social cognition (Eitam \& Higgins, 2010), but it has not always been 
defined in a clear manner. Upon close examination of the literature, goal relevance seems to cover at least three dissociable meanings. (i) Goal relevance is sometimes used interchangeably with task relevance (e.g., Folk \& Remington, 2008). A stimulus is goal-relevant if it allows implementation of a specific goal (e.g., if a task is to press a left button for red targets, red targets become goal-relevant in this sense). (ii) Alternatively, goal relevance may refer to the degree to which a stimulus is informative about the satisfaction status of pursued goals (see Moors, 2007). A stimulus is more or less informative when the information it conveys about the satisfaction status of goals is more or less certain or reliable. Therefore, goal relevance in this sense shares similarities with notions of certainty or precision, and with reliability and metacognitive confidence (Gibbons, Schnuerch, \& Stahl, 2016; Pfabigan, Zeiler, Lamm, \& Sailer, 2014). (iii) Finally, goal relevance may correspond to the impact a stimulus has on the individual's goals (Roseman \& Smith, 2001). A stimulus is deemed more or less relevant if its impact on the goal is larger or smaller. For instance, winning 20 Euro has a larger impact on the goal to win money than winning only 2 Euro $^{1}$. Feedback on task performance can in some cases be relevant in the first sense (e.g., when the participant is asked to make responses dependent on the feedback), but in most standard cases, feedback is relevant in the second and/or the third sense. Feedback comes with a degree of informativeness, certainty or reliability, and it can impact goals to a large or small extent.

These three meanings of goal relevance are partly but not entirely dissociable. A stimulus that is goal-relevant in the first sense (allowing implementation of a goal) signals potential goal satisfaction, and in this way, it is also relevant in the second (reliability) and the third sense (impact). However, stimuli can be relevant in the second and/or third sense without being relevant in the first sense. For instance, the presentation of a reward has an impact on one's goal to win money (cf. third sense) but may not allow implementing a further goal (cf. first sense). Goal relevance in the second and third sense are not entirely

\footnotetext{
${ }^{1}$ Impact has partial but not complete overlap with the economical definition of value (Holroyd \& Coles, 2002; Sambrook \& Goslin, 2015; Walsh \& Anderson, 2012). Value can be considered as a combination of impact and valence: A gain of 20 Euro has more value than a gain of 2 Euro, which has more value than a loss of 2 Euro, which in turn has more value than a loss of 20 Euro. A loss of 20 Euro has the same impact as a gain of 20 Euro, and a loss of 2 Euro has the same impact as a gain of 2 Euro. Losses and gains of 20 Euro have a higher impact than losses and gains of 2 Euro.
} 
independent either. It seems reasonable to assume that feedback has to have a minimal degree of reliability before it can impact on a person's goals, and that increasing the reliability of the feedback increases its sweak to very strong.

In the present study, we sought to test the assumption that a feedback's goal relevance in the second sense (reliability) can account for amplitude variations at the level of the FRN component during PM, besides or beyond what valence alone or expectedness alone can explain. To this end, we used a specific experimental setting in which the informativeness or reliability of feedback stimuli was varied independently from their valence and their degree of expectedness ${ }^{2}$. In addition, we made sure to keep the task relevance (goal relevance in the first sense) of the feedback stimuli constant. Given the abovementioned potential influence of goal relevance in the second sense (informativeness) on goal relevance in the third sense (impact), we could not isolate the former from the latter. Thus, the present study sought to investigate the role of goal relevance in the second (and/or third) sense on the FRN component. From now on, we use the term 'goal relevance' in the second sense unless specified otherwise. We manipulated the goal relevance of feedback systematically across blocks using a stringent within-subjects experimental design (see also Gibbons et al., 2016; Pfabigan et al., 2014).

Participants performed a standard speeded Go/NoGo task, as used previously and validated by Aarts and Pourtois (2010; 2012), Koban, Pourtois, Bediou, and Vuilleumier (2012), and Vocat, Pourtois, and Vuilleumier (2008). The goal of this task is to respond both accurately and timely (before an arbitrary reaction time speed cut-off unknown to the participants). On each and every trial, feedback regarding performance is provided to the participant. Because reaction time (RT) is inherently more variable/labile and harder to decipher than accuracy for participants, they have to carefully monitor the feedback in order

\footnotetext{
${ }^{2}$ Whether a stimulus is positive or negative is independent of whether it provides an opportunity for goal implementation (goal relevance in the first sense) and whether or not it is informative about goal satisfaction (goal relevance in the second sense). Also, positive and negative stimuli may both have an impact on goal satisfaction (goal relevance in the third sense), although the phenomenon of loss aversion (Kahneman \& Tversky, 1984) suggests that losses have a larger impact on goals than equal wins. Whether a stimulus is expected or unexpected is independent of goal relevance in all three senses: expected and unexpected stimuli provide equal opportunity for the implementation of goals, they can be equally informative about the satisfaction status of goals, and their impact on goal satisfaction may be equally high.
} 
to assess whether their actions are goal-conducive (i.e., correct and fast enough) or not (i.e., either incorrect or too slow). Crucially, across blocks, participants were told that this feedback was either relevant (reflecting their behavior and therefore informative about the satisfaction status of their goals) or irrelevant. Goal relevance in the first sense was kept constant by making the feedback equally taskrelevant in both contexts: Participants had to discriminate the emotional content of the feedback now and then, equally often in both contexts. Hence, even when the feedback was irrelevant, participants still had to attend to it in order to carry out the additional emotion discrimination task.

We predicted a significant interaction effect between feedback valence (positive vs. negative) and context (relevant vs. irrelevant) at the FRN level, consistent with the goal relevance account. More specifically, we reckoned that the FRN should capture the difference between positive and negative feedback in the relevant context only, while no such valence effect should be observed in the irrelevant context. In the irrelevant context, the rapid and seemingly automatic processing of feedback valence (and expectedness) at the FRN level should be transiently interrupted (see Moors, 2007; Ullsperger et al., 2014b). Obviously, this does not imply that PM does not operate in the irrelevant context. PM can flexibly sreliable external cues in the irrelevant context may therefore lead to a shift from external to internal monitoring. In other words, we hypothesized an enhanced internal monitoring in the irrelevant compared to the relevant context, which would be expressed by a larger Correct-Related Negativity (CRN) differentiation between slow and fast hits in this condition specifically.

Experiment 1 used a procedure in which reward probability was low in the relevant context. That is, participants were more likely to receive negative than positive feedback given the specifics of the speeded Go/NoGo task used (see Aarts \& Pourtois, 2012). No such asymmetry was implemented in the irrelevant context, however, where feedback valence was unrelated to task performance, and hence positive and negative feedback were shown equally often in a random order. Obviously, this difference created a potential confound in terms of objective reward probability, and therefore most likely also in terms of subjective reward expectation, also called reward expectancy (see Ferdinand et al., 2012; von Borries et al., 2013). In the current study, we used the term 'expectancy' to refer to the manipulation of 
feedback probability. Thus, in Experiment 1 the two main contexts differed not only regarding goal relevance per se, but also regarding reward expectancy (which was lower in the relevant than the irrelevant context), a factor which is known to influence the magnitude of the FRN as well (Ferdinand et al., 2012; Holroyd et al., 2008). In order to overcome this limitation, we ran Experiment 2, in which we used the same task in a new sample of participants, but we set up the experimental procedure (i.e., the response deadline) in such a way that the number of positive vs. negative feedback stimuli was matched between the relevant and irrelevant context. As a result, only goal relevance (but not reward expectancy) varied systematically between both contexts. In sum, goal relevance was manipulated in each of the two experiments, but its relation with reward expectancy differed across them.

\section{Methods}

\subsection{Participants}

In Experiment 1, thirty five healthy adult subjects participated in exchange of 30 Euro compensation. Five subjects had to be excluded from the subsequent analyses: one based on a strict age criterion (i.e., this participant's age was 3 SDs above the mean age of the sample), two due to a failure in the experimental procedure, and two due to the excessive noise and artifacts during the EEG recording. Hence, the final sample consisted of 30 subjects (6 men; mean age: 21.9; $\mathrm{SD}=2.7)$. All subjects were right-handed and had normal or corrected-to-normal vision. They were free of neurological or psychiatric history and of any psychoactive medication. Prior to the experiment they gave written informed consent.

In Experiment 2, forty subjects participated in exchange of 30 Euro compensation. Ten subjects had to be excluded from the subsequent analyses: three due to the excessive noise and artifacts during the EEG recording, and seven due to an insufficient number (3 SDs below the sample mean) of fast hits necessary for further analyses. Hence, the final sample consisted of 30 subjects ( 8 men; mean age: 22.1; $\mathrm{SD}=2.3)$

As anxiety and perceived locus of control can each influence PM at the FRN level (see Aarts \& Pourtois, 2012), we administrated the STAI-trait version (Dutch version; Defares, van der Ploeg, \& 
Spielberger, 1979), and the Locus of Control (LOC) questionnaire (Dutch version; Rotter, 1966) in both experiments. For participants from Experiment 1, the mean STAI score was 39.2 (range: 25-62) and the LOC score 13 (range: 5-18). In Experiment 2, the mean trait anxiety level was 37.3 (range: 23-68) and the mean LOC score 11.7 (range: 3-19). Independent samples t-tests confirmed that there were no significant differences in the level of trait anxiety $\left(t_{(58)}=0.73, p=0.472\right)$ and locus of control $\left(t_{(58)}=1.31, p=0.198\right)$ between the two experiments.

2.2. Experimental paradigm and procedure

A modified version of a speeded Go/NoGo task was used in both experiments (Aarts \& Pourtois, 2010; 2012; Koban et al., 2012; Pourtois, 2011; Vocat et al., 2008). The experimental design is presented in Figure 1.

[insert Figure 1 here]

All visual stimuli were shown on a 21 -inch CRT screen. Each trial started with a black fixation cross lasting for $1000 \mathrm{~ms}$. Then, a black arrow ('cue'), either oriented up or down, was presented. After a variable interval ranging from $1000 \mathrm{~ms}$ up to $2000 \mathrm{~ms}$, the black arrow became either green or turquoise, while its orientation could either remain identical or shift in the opposite (in-plane) orientation. When the black arrow turned green and the orientation remained unchanged ('target'), participants were instructed to press a predefined key on the response box as fast as possible with the index finger of their right hand ('Go trials'). However, participants had to withhold responding when either the arrow became green but flipped orientation, or when the arrow became turquoise and kept its initial orientation ('non-targets' in 'NoGo trials'). In the absence of motor responses, targets and non-targets remained on the screen for 1000 ms. Cues, targets, and non-targets consisted of an arrow $\left(11.4^{\circ} \times 0.05^{\circ}\right.$ of visual angle at a $50 \mathrm{~cm}$ viewing distance), presented in the center of the screen on a white background. After motor responses (correct: 'hits'; incorrect: 'false alarms'), a colored frame was presented for $1000 \mathrm{~ms}$ around the target. Following that, a response-feedback interval was presented for $1000 \mathrm{~ms}$. This event consisted of the presentation of 
the colored frame only, and it served to indicate whether the feedback provided was relevant or not (see below). The colored frame remained on the screen around the feedback for $1000 \mathrm{~ms}$ (see also Figure 1).

Participants were given positive feedback when they responded both correctly and fast to Go trials ('fast hit'), and when they correctly withheld responding to NoGo trials ('correct inhibition'). They were given negative feedback when the response was correct but too slow ('slow hit'), when they gave a response to NoGo trials ('false alarm'), or when there was no response to Go trials ('omission'). We used an online adaptive algorithm to set up a limit for correct and fast RTs (i.e., response deadline procedure) in Go trials. At the beginning of the experiment, the RT limit was set to $300 \mathrm{~ms}$ (based on previous pilot testing; Vocat et al., 2008). This limit was adjusted online (i.e., after each trial) as a function of the immediately preceding trial history, more specifically, as the mean of current and previous RTs. Responses that were slower than the limit were classified as slow hits; responses that were faster than the limit were classified as fast hits. The advantage of this algorithm is that uncertainty about current RTs is high throughout the task (given the fluctuations of RTs), which motivates participants to actively attend to the external feedback stimulus presented after each response to infer whether their actions (during Go trials) were timely (fast hits) or not (slow hits). Moreover, the response deadline is updated throughout the experiment in order to avoid habituation or fatigue, and it is set up in such a way that correct and fast responding to Go trials is fairly difficult to achieve (Aarts \& Pourtois, 2010; 2012; Dhar \& Pourtois, 2011; Dhar, Pourtois, \& Wiersma, 2011; Koban, Pourtois, Vocat, \& Vuilleumier, 2010; Koban et al., 2012; Vocat et al., 2008). This ensures that participants show a high involvement in the task (Atkinson \& Feather, 1966). Feedback following actions on NoGo trials (positive feedback following a correct inhibition or negative feedback following a false alarm) was not informative as participants could readily evaluate the accuracy of their actions on these trials using internal monitoring (see Koban et al., 2012 for a clear demonstration using a similar task). Therefore, in this study, we focused on the ERP responses to evaluative and highly informative feedback following fast or slow hits to Go trials only.

As the main goal of our study was to assess the role of feedback relevance (reliability) for eliciting a clear reward prediction error signal during PM, we created two contexts (relevant vs. irrelevant) varying 
with respect to the relationship between the response and the given feedback, while the task and the stimuli remained identical in both contexts. This manipulation was always clearly indicated to participants by the use of: (i) written instructions delivered before the start of each block, (ii) a colored frame (either blue or magenta) appearing immediately around targets or non-targets after the manual (key press) response and remaining on the screen until feedback offset throughout the whole block (see above and Figure 1). In the relevant condition, the feedback was reliable in the sense of being always related to the actual speed and the accuracy of the response. Therefore, participants always received positive feedback after fast hits and correct inhibitions, while they received negative feedback in case of slow hits, false alarms, and omissions. In contrast, in the irrelevant condition, the feedback provided after the response was not related to the actual performance. Positive and negative feedback were delivered randomly (drawn from a pre-existing list), with an equal probability of these two valences, irrespective of the accuracy and speed of the preceding action.

Feedback stimuli consisted of emotional or neutral faces. The feedback was always uninformative in the irrelevant condition and always informative in the relevant condition. Thus, participants were less encouraged to pay attention to the feedback in the irrelevant than the relevant condition. To avoid unequal attention allocation to the feedback stimulus in both conditions, we included 'catch trials': In $17 \%$ of the trials and in random order, participants were additionally asked (second task) to categorize the valence of the face presented as feedback. At the offset of the feedback, a question probed their ability to categorize the face as carrying a positive vs. negative expression. For this purpose, they were instructed to press predefined keys on the response box with the index finger of their right hand. No time limit was imposed and accuracy was emphasized.

The experiment consisted of a training session with 32 trials (always with a relevant feedback), followed by 6 experimental blocks, each including 56 trials (40 Go and 16 NoGo trials in each block). Go/NoGo trial presentation was randomized within blocks. There were 3 blocks per condition (relevant vs. irrelevant). Hence, feedback relevance served as a within-subjects factor in subsequent statistical analyses. Two specific orders were created: R-I-R-I-R-I or I-R-I-R-I-R, with 'R' referring to relevant 
context, and 'I' to irrelevant context. Moreover, the mapping of frame color (blue vs. magenta) on context (relevant vs. irrelevant) was counterbalanced across participants. Hence, in total, four different versions of the experimental procedure were created, and participants were randomly assigned to one of them at the beginning of the experiment.

After each block, feedback relevance was evaluated by means of a Visual Analog Scale (VAS). More specifically, participants were asked to rate how relevant (for gauging task performance) feedback was during the previous block on a continuous scale ranging from -50 (not at all) to +50 (very much). These subjective ratings served as a direct manipulation check of feedback relevance.

In Experiment 1 the online adjustment and calibration of the RT speed limit used to categorize Go trials into fast vs. slow hits was similar to previous studies (see Aarts \& Pourtois, 2012). In Experiment 2, task demands and trial structure were identical to Experiment 1, but we modified the procedure with the aim of achieving an equal amount of positive and negative feedback in the relevant context (for a majority of participants, and despite the inter-individual variability in their RTs). Therefore, we adjusted the RT deadline across blocks using specific (predefined) time limits. After extensive piloting, we found out that pre-setting the time limit to 300 (Blocks 1-2), 275 (Blocks 3-4), and 250 ms (Blocks 5-6) yielded the expected effect (i.e., a balanced number of fast hits followed by positive feedback, and slow hits followed by negative feedback) in the majority of subjects. With these parameters, we also dealt with unspecific learning/habituation effects. Participants were never informed about this procedure. Stimulus presentation and response recording were controlled using E-prime software (V2.0., http://www.pstnet.com/products/eprime/).

\subsection{Face stimuli}

In both experiments the same set of 24 different face identities (12 per gender) with a neutral, happy or angry emotional expression were selected from the Karolinska Directed Emotional Faces database (KDEF; Lundqvist, Flykt, \& Öhman, 1998). In one group ( $n=15), 12$ happy and 12 neutral faces were used in the relevant condition, and another 12 happy and 12 neutral expressions in the irrelevant context. 
In this group, the happy faces served as the positive feedback stimuli and the neutral faces as the negative feedback stimuli. In the other group $(n=15), 12$ angry and 12 neutral faces were used as feedback stimuli in the relevant condition, and another 12 angry and 12 neutral expressions in the irrelevant feedback condition. In this group, the neutral faces served as positive feedback stimuli and the angry faces as negative feedback stimuli. For each participant, the actual faces used as positive or negative feedback were selected randomly from the set of 24 faces. We made sure that each face was presented equally often in the relevant and the irrelevant condition. For each participant and condition separately, 8 faces ( 4 per gender and 4 per emotional content) were preselected and used for the catch trials (see above).

After the experimental session, all the faces used during the experiment $(n=48)$ were presented again one by one (without time limit) with their corresponding colored frame. Participants were asked to rate the valence of each face on a scale ranging from -50 (very negative) to +50 (very positive).

There were several reasons for using emotional faces as feedback stimuli instead of binary symbolic stimuli (e.g., the written words: correct and incorrect, or green and red dots). First, the use of faces as feedback was previously validated (the procedure used in the relevant condition was identical to the one used previously by Aarts \& Pourtois, 2012). Second, the use of faces allowed us to make the content of the feedback stimuli unpredictable (we used 24 different face identities, presented in a random order, which resulted in low statistical regularity), in order to encourage their thorough exploration by the participant each time. This would not be possible with binary symbolic stimuli. Further, to make sure that a thorough processing of the positive vs. negative content of the stimuli took place in both contexts (relevant and irrelevant), we added catch trials in which participants had to respond on the basis of this content. This would be more difficult to achieve with simple symbolic stimuli. Finally, emotional faces provide potent social stimuli that may have a stronger impact than symbolic stimuli (although this argument awaits further empirical validation). 


\subsection{EEG acquisition and processing}

In both experiments, participants were seated in a dimly lit, sound-attenuated, and electrically shielded cabin. Continuous EEG was acquired at $512 \mathrm{~Hz}$ using a 64-channel (pin-type) Biosemi Active Two system (http://www.biosemi.com), referenced online to the Common ModeSense (CMS)-Driven Right Leg (DRL) ground. All electrodes were placed according to the extended International 10-20 EEG system using an elastic head cap. The horizontal and vertical EOG were monitored by means of 4 electrodes, placed above and below the right eye and on the outer canthi of both eyes.

\subsubsection{ERP analyses}

ERPs of interest were computed offline following a standard sequence of data transformations (Keil, Debener, Gratton, Junghöfer, Kappenman, Luck, Luu, Miller, \& Yee, 2014): (i) 50-Hz notch filter; (ii) rereferencing of the EEG signal using a common average reference; (iii) -500/+1000 ms segmentation around the onset of the feedback stimulus, or $-500 /+500 \mathrm{~ms}$ segmentation around the response onset; (iv) pre-stimulus interval baseline correction (from $-500 \mathrm{~ms}$ to feedback onset), or pre-response interval baseline correction (from -500 to $-300 \mathrm{~ms}$ prior to the motor response); (v) vertical ocular correction for blinks (Gratton, Coles, \& Donchin, 1983); (vi) semi-automatic artifact rejection (trials with motor artifacts were rejected, with a fixed criterion of $\pm 80 \mu \mathrm{V}$ ); (vii) averaging of the feedback-locked ERPs for each type of feedback separately (i.e., positive feedback following fast hits and negative feedback following slow hits in the relevant condition, positive and negative feedback following hits in the irrelevant condition), or averaging of the response-locked ERPs for each type of response separately (fast hits and slow hits recorded in the relevant and irrelevant conditions); and (viii) low pass digital filtering of the individual average data $(30 \mathrm{~Hz})$.

We focused on the Feedback-Related Negativity (FRN) and the P3a and P3b components, which all have been related to feedback processing in previous ERP studies focused on PM (Aarts \& Pourtois, 2012; Bismark, Hajcak, Whitworth, \& Allen, 2013; Ferdinand et al., 2012; Fischer \& Ullsperger, 2013; Pfabigan et al., 2011; 2014; von Borries et al., 2013; Walsh \& Anderson, 2012). The FRN was defined as 
the mean voltage within $250-300 \mathrm{~ms}$ after feedback onset over frontal and fronto-central electrodes along the midline ( $\mathrm{Fz}$ and $\mathrm{FCz}$ pooled together). P3a was defined as the mean voltage appearing 350-450 ms after feedback onset at the same locations as the FRN. The P3b amplitude was measured as a mean voltage between 400 and $600 \mathrm{~ms}$ after feedback onset at a cluster of centro-parietal electrodes $(\mathrm{CPz}, \mathrm{Pz}$, P1, and P2 pooled together). Additionally, the CRN (in response to either fast or slow hits) was defined as the mean voltage recorded $10 \mathrm{~ms}$ prior to until $30 \mathrm{~ms}$ after motor response at fronto-central electrodes along the midline (Fz and $\mathrm{FCz}$ pooled together).

\subsubsection{Spatio-temporal separation of FRN and P3a}

To further validate the presence of two successive and distinctive neural events during feedback processing (i.e., FRN and P3a) and given their partial spatial overlap when titrated using a standard peak analysis (see Holroyd et al., 2008), we performed an auxiliary ERP topographical mapping analysis, using the Cartool program (http://brainmapping.unige.ch/cartool). While the previous analysis focused on amplitude variations occurring at electrodes $\mathrm{Fz}$ and $\mathrm{FCz}$ for the FRN and P3a as a function of feedback valence and relevance, we wanted to establish more formally that these two successive feedback-locked ERP components could indeed be dissociated from one another based on their topographical properties (and hence when considering the entire electric field composed of 64 channels concurrently; for a similar approach see Pourtois, De Pretto, Hauert, \& Vuilleumier, 2006).

Following a standard procedure (see Pourtois, Delplanque, Michel, \& Vuilleumier, 2008; Murray, Brunet, \& Michel, 2008), the grand average ERP waveforms were submitted to a K-means clustering algorithm that enabled isolating the underlying dominant topographical maps. This algorithm clearly revealed the presence of two dissociable topographical components during the 200-500 ms post-feedback onset interval, one corresponding to the FRN (see Figure 2B) and the other one to the P3a (see Figure 2C). The spatial correlation between these two ERP topographical maps was .63 and .84 for Experiment 1 and 2 , respectively. 


\subsection{Statistical analyses}

For each experiment separately, we used mixed model ANOVAs to analyze the behavioral and electrophysiological data. These ANOVAs included the within-subjects factors VALENCE (positive vs. negative) and CONTEXT (relevant vs. irrelevant) for feedback-related ERPs, while for response-related ERPs, RESPONSE (fast hit vs. slow hit) and CONTEXT were used as within-subjects variables. Significant main or interaction effects were reported first, followed by post hoc paired t-tests when applicable.

For the feedback-related electrophysiological data, we analyzed the differential (and global) processing of positive vs. negative feedback at the ERP level (as a function of the perceived feedback relevance) irrespective of the actual facial expressions used to convey these two feedbacks. To this aim, the neutral faces used as negative feedback in one group of participants were pooled together with the angry faces also used as negative feedback in the other group of participants. Similarly, neutral faces used as positive feedback in this latter group were combined with happy faces used as positive feedback in the former group. This way, the observed ERP effects were devoid of pre-existing physical or emotional differences between the two categories (positive vs. negative feedback).

\section{Results}

\subsection{Behavioral results}

Accuracy for catch trials was high and comparable in both contexts and experiments (see Table 1). In Experiment 1 participants' actual performance was balanced between the relevant and irrelevant context (all $p s>0.65$, see Table 1), suggesting a comparable task engagement in these two conditions. As expected, participants had a larger number of slow hits (approximately $2 / 3$ ) than fast hits $(1 / 3)$, both in the relevant $\left(t_{(29)}=-7.26, p<0.001\right)$ and the irrelevant context $\left(t_{(29)}=-8.44, p<0.001\right)$. Despite this asymmetry at the response level, the number of positive vs. negative feedback stimuli delivered in the irrelevant context was not statistically different $\left(t_{(29)}=0.22, p=0.828\right)$, confirming that feedback valence 
and response (speed) were properly decoupled in this context, unlike the relevant context, where the number of negative and positive feedback stimuli differed significantly $\left(t_{(29)}=-7.26, p<0.001\right.$; see Table 1). RTs were overall longer in the irrelevant compared to the relevant context, however, irrespective of the actual action being performed (fast hits, $t_{(29)}=-5.32, p<0.001$; slow hits, $t_{(29)}=-3.14, p=0.004$; false alarms, $t_{(29)}=-3.31, p=0.003$ ), suggesting a global change (i.e., slowing down effect with irrelevant feedback) in PM processes created by the context manipulation, as opposed to specific alterations (e.g., systematic change of the speed-accuracy tradeoff).

In Experiment 2, participants' performance was similarly balanced between the two contexts (all $p s>0.55$, see Table 1). Importantly, because of the change in the experimental procedure made in this experiment (see above), the number of positive and negative feedback stimuli delivered in each context was similar (CONTEXT x VALENCE interaction: $F_{(1,29)}=0.72, p=0.401, \eta_{p}{ }^{2}=0.012$ ). Hence, there was no asymmetry between positive and negative feedback, neither in the relevant $\left(t_{(29)}=-0.89, p=0.379\right)$ nor in the irrelevant context $\left(t_{(29)}=-0.33, p=0.974\right)$. Similarly to Experiment 1 , longer RTs were observed in the irrelevant compared to the relevant context in this experiment, irrespective of the actual type of response given (for fast hits, $t_{(29)}=-5.76, p<0.001$; for slow hits, $t_{(29)}=-4.73, p<0.001$; and for false alarms, $\left.t_{(29)}=-2.41, p=0.023\right)$.

\section{[insert Table 1 here]}

\subsection{Evaluation of feedback relevance}

In Experiment 1, the data of one subject were lost, and they were replaced by the corresponding conditionspecific mean values calculated for the whole sample. Importantly, feedback provided in the relevant context $(M=11.31$, SEM $=4.38)$ was evaluated as more reliable when compared with the irrelevant condition $(M=-12.38, \mathrm{SEM}=3.13)\left(F_{(1,29)}=18.63, p<0.001, \eta_{p}{ }^{2}=0.391\right)$ confirming that the manipulation of goal relevance across successive blocks was successful. 
In Experiment 2 feedback provided in the relevant context $(M=30.81, \mathrm{SEM}=2.61)$ was also evaluated as more reliable compared to feedback given in the irrelevant one $(M=-18.91, \mathrm{SEM}=2.97)$, as confirmed by a highly significant main effect of $\operatorname{CONTEXT~}\left(F_{(1,29)}=118.92, p<0.001, \eta_{p}{ }^{2}=0.804\right)$.

A direct comparison of these ratings between the two experiments showed a significant EXPERIMENT $\times$ CONTEXT interaction $\left(F_{(2,58)}=13.31, p=0.001, \eta_{p}{ }^{2}=0.187\right)$, indicating that participants judged the feedback to be more reliable in Experiment 2 than in Experiment 1. Follow-up ttests showed that for the relevant context only $\left(t_{(58)}=-3.82, p<0.001\right)$ feedback was judged as more reliable in Experiment 2 compared to Experiment 1. No such difference was observed in the irrelevant context $\left(t_{(58)}=1.51, p=0.136\right)$.

\subsection{Ratings of the perceived valence of faces}

In Experiment 1, the data of one subject were lost and replaced by the mean values calculated for the whole sample for these specific conditions. As expected, faces used as positive feedback stimuli were rated as more positive $(M=20.61, \mathrm{SEM}=1.75)$ than faces used as negative feedback stimuli $(M=-25.37$, $\mathrm{SEM}=1.51)\left(t_{(59)}=24.91, p<0.001\right)$. This valence effect was balanced across the two contexts $\left(t_{(29)}=\right.$ $1.67, p=0.105$ for positive and $t_{(29)}=0.75, p=0.459$ for negative feedback).

Face rating data of Experiment 2 were similar to those of Experiment 1. Faces used as positive feedback stimuli were rated as more positive $(M=18.47$, SEM $=1.94)$ than faces used as negative feedback stimuli $(M=-24.48, \mathrm{SEM}=1.87)\left(t_{(59)}=28.68, p<0.001\right)$. This effect was the same for both contexts $\left(t_{(29)}=1.89, p=0.168\right.$ for positive and $t_{(29)}=-0.99, p=0.327$ for negative feedback $)$.

\subsection{Electrophysiological results}

\subsubsection{FRN component}

In Experiment 1, for amplitude values recorded from $\mathrm{Fz}$ and $\mathrm{FCz}$ electrodes pooled together 250-300 ms post-face stimulus onset, the main effect of CONTEXT was significant $\left(F_{(1,29)}=6.89, p=0.014, \eta_{p}{ }^{2}=\right.$ 0.192), while the main effect of VALENCE was not $\left(F_{(1,29)}=1.09, p=0.304, \eta_{p}{ }^{2}=0.036\right)$. Importantly, a 
significant CONTEXT x VALENCE interaction was found $\left(F_{(1,29)}=5.49, p=0.026, \eta_{p}{ }^{2}=0.159\right)$. More negative amplitude values were recorded for negative $(M=-1.35 \mu \mathrm{V}, \mathrm{SD}=2.71)$ compared to positive feedback $(M=0.22 \mu \mathrm{V}, \mathrm{SD}=2.73)$. However, this valence effect was found in the relevant context only $\left(t_{(29)}=2.08, p=0.047\right)$. No such effect was visible when the feedback was deemed irrelevant $\left(t_{(29)}=-0.98\right.$, $p=0.331)$, with $M=-1.35 \mu \mathrm{V}(\mathrm{SD}=2.75)$ and $M=-1.37 \mu \mathrm{V}(\mathrm{SD}=2.73)$ in response to negative and positive feedback, respectively.

In Experiment 2, the main effect of CONTEXT was significant $\left(F_{(1,29)}=4.57, p=0.041, \eta_{p}{ }^{2}=\right.$ 0.136), showing that feedback (regardless of its valence) elicited a larger negative component in the irrelevant compared to the relevant context (very much like the FRN recorded in Experiment 1), suggesting a differential (valence-unspecific) processing of the feedback in these two contexts. Moreover, neither the main effect of VALENCE $\left(F_{(1,29)}=1.35, p=0.255, \eta_{p}{ }^{2}=0.044\right)$, nor the CONTEXT $\mathrm{x}$ VALENCE interaction $\left(F_{(1,29)}=1.81, p=0.189, \eta_{p}{ }^{2}=0.059\right)$ reached significance, and only a trend towards more negative amplitudes was found $\left(t_{(29)}=1.64, p=0.112\right)$ for negative $(M=-2.44 \mu \mathrm{V}, \mathrm{SD}=$ 2.39) compared to positive feedback $(M=-1.89 \mu \mathrm{V}, \mathrm{SD}=2.31)$ in the relevant context. No such trend $\left(t_{(29)}=0.02, p=0.988\right)$ was visible in the irrelevant context $(M=-2.63 \mu \mathrm{V}, \mathrm{SD}=1.94$, and $M=-2.63 \mu \mathrm{V}$, $\mathrm{SD}=2.41$ for negative and positive feedback, respectively $)^{3}$.

[insert Figures 2 and 3 here]

\subsubsection{P3a component}

In Experiment 1, the early valence effect (in the relevant context) extended beyond the time-course of the FRN (250-300 ms), and yielded a conspicuous P3a component (350-450 ms), likely caused by the

\footnotetext{
${ }^{3}$ Visual inspection of the waveforms presented in Figures 2 and 3 suggested that a frontal P2 component occurred prior to the FRN component. This positive component is the frontal counterpart of the occipito-temporal facespecific N170 component, usually known in the ERP literature as the VPP (Vertex Positive Potential; see Jeffreys \& Tukmachi, 1992; Jeffreys, 1993). To exclude the possibility of any earlier context- or valence-specific effect at this level (P2), an auxiliary analysis was run. To analyze the VPP, we extracted the mean amplitude of the ERP signal during the 160-200 ms post-stimulus onset interval at electrodes Fz and FCz (collapsed together). The 2 x 2 ANOVA (with CONTEXT and VALENCE as within-subjects factors) did not reveal any significant main or interaction effects (at a standard $p<.05$ value) however, for none of the two experiments, confirming that PM processes (at the feedback level) were not affected by these factors prior to onset of the FRN component.
} 
infrequent presentation of positive feedback in this context. No such orienting response was found in the irrelevant context where the amount of positive and negative feedback was balanced (see Figure 2). These observations were verified by significant main effects of $\operatorname{CONTEXT}\left(F_{(1,29)}=19.21, p<0.001, \eta_{p}{ }^{2}=\right.$ $0.398)$ and VALENCE $\left(F_{(1,29)}=10.05, p=0.004, \eta_{p}{ }^{2}=0.257\right)$, and a significant interaction effect between CONTEXT and VALENCE $\left(F_{(1,29)}=7.97, p=0.008, \eta_{p}{ }^{2}=0.216\right)$. A P3a component was elicited for positive $(M=2.09 \mu \mathrm{V}, \mathrm{SD}=2.84)$ but not negative feedback $(M=0.16 \mu \mathrm{V}, \mathrm{SD}=2.45)$ in the relevant condition $\left(t_{(29)}=3.62, p=0.001\right)$. No such oddball-like effect was evidenced in the irrelevant condition $\left(t_{(29)}=0.18, p=0.859\right)$, with a negative and balanced amplitude for both feedback valences $(M=-0.78$ $\mu \mathrm{V}, \mathrm{SD}=1.48$ for negative feedback; $M=-0.48 \mu \mathrm{V}, \mathrm{SD}=1.96$ for positive feedback).

In Experiment 2, only the main effect of CONTEXT was significant $\left(F_{(1,29)}=19.68, p<0.001, \eta_{p}{ }^{2}\right.$ $=0.404)$, while the main effect of $\operatorname{VALENCE}\left(F_{(1,29)}=0.47, p=0.5, \eta_{p}{ }^{2}=0.016\right)$, and the interaction effect between CONTEXT and VALENCE $\left(F_{(1,29)}=2.47, p=0.127, \eta_{p}{ }^{2}=0.078\right)$ were non-significant. The P3a amplitude was less negative for feedback (positive and negative pooled together) shown in the relevant $(M=-0.62 \mu \mathrm{V}, \mathrm{SD}=2.71)$ compared to the irrelevant context $(M=-1.95 \mu \mathrm{V}, \mathrm{SD}=2.02)$.

\subsubsection{P3b component}

In Experiment 1, at a later time following feedback onset (400-600 ms), a large P3b component was elicited at posterior parietal sites, especially for relevant compared to irrelevant feedback. This observation was confirmed by a significant main effect of $\operatorname{CONTEXT~}\left(F_{(1,29)}=14.28, p=0.001, \eta_{p}{ }^{2}=0.432\right)$, while the main effect of VALENCE $\left(F_{(1,29)}=0.24, p=0.627, \eta_{p}{ }^{2}=0.013\right)$ and the CONTEXT x VALENCE interaction $\left(F_{(1,29)}=0.32, p=0.438, \eta_{p}{ }^{2}=0.017\right)$ were not significant. As it can be seen from Figure 4, this P3b component had a larger amplitude for feedback delivered in the relevant $(M=3.85 \mu \mathrm{V}, \mathrm{SD}=2.79 ; M$ $=3.82 \mu \mathrm{V}, \mathrm{SD}=2.81$ for positive and negative feedback, respectively) compared to the irrelevant context $(M=2.55 \mu \mathrm{V}, \mathrm{SD}=2.93 ; M=2.88 \mu \mathrm{V}, \mathrm{SD}=2.23$ for positive and negative feedback, respectively $)$, regardless of feedback valence $\left(t_{(29)}=0.11, p=0.876\right.$ and $t_{(29)}=-0.93, p=0.572$ for the relevant and irrelevant context, respectively). 
Similarly to Experiment 1, in Experiment 2 feedback shown in the relevant context led to a much larger P3b component compared with the irrelevant context, as captured by a significant main effect of $\operatorname{CONTEXT}\left(F_{(1,29)}=27.67, p<0.001, \eta_{p}{ }^{2}=0.526\right)$. However, unlike what was found in Experiment 1 , where this effect was valence-unspecific, here the valence of the feedback did influence the amplitude of the P3b component, in the relevant context selectively, as shown by a significant CONTEXT $\mathrm{x}$ VALENCE interaction effect $\left(F_{(1,29)}=9.29, p=0.009, \eta_{p}{ }^{2}=0.317\right)$. More specifically, in the relevant context, the P3b had a larger amplitude for negative $(M=4.28 \mu \mathrm{V}, \mathrm{SD}=2.33)$ compared to positive feedback $\left(M=3.33 \mu \mathrm{V}, \mathrm{SD}=2.27 ; t_{(29)}=-2.28, p=0.021\right)$, while no such clear amplitude difference depending on feedback valence was seen in the irrelevant context $(M=1.98 \mu \mathrm{V}, \mathrm{SD}=1.75 ; M=1.83 \mu \mathrm{V}$, $\mathrm{SD}=1.16$ for negative and positive feedback, respectively; $t_{(29)}=1.11, p=0.272$ ).

\section{[insert Figure 4 here]}

\subsubsection{CRN component}

In Experiment 1, the amplitude difference at the CRN level between slow hits and fast hits was larger in the irrelevant than the relevant condition (see Figure 5). The ANOVA revealed a non-significant main effect of CONTEXT $\left(F_{(1,29)}=0.14, p=0.713, \eta_{p}{ }^{2}=0.005\right)$, a trend-significant main effect of RESPONSE $\left(F_{(1,29)}=3.86, p=0.059, \eta_{p}{ }^{2}=0.117\right)$, and importantly, a significant CONTEXT x RESPONSE interaction $\left(F_{(1,29)}=4.66, p=0.039, \eta_{p}{ }^{2}=0.138\right)$. Follow-up paired t-tests confirmed a larger CRN for slow $(M=-$ $4.18 \mu \mathrm{V}, \mathrm{SD}=3.62)$ than fast hits $(M=-3.12 \mu \mathrm{V}, \mathrm{SD}=3.56)$ in the irrelevant context only $\left(t_{(29)}=2.56, p\right.$ $=0.016$ ), with no such early (response-locked) differentiation in the relevant context (with $M=-4.04 \mu \mathrm{V}$, $\mathrm{SD}=3.65$, and $M=-3.74 \mu \mathrm{V}, \mathrm{SD}=3.67$ for slow and fast responses, respectively; $t_{(29)}=1.02, p=0.317$ ).

In Experiment 2, the main effect of CONTEXT was found to be non-significant $\left(F_{(1,29)}=0.04, p=\right.$ $\left.0.836, \eta_{p}{ }^{2}=0.002\right)$, while the main effect of RESPONSE was highly significant $\left(F_{(1,29)}=13.68, p=0.001\right.$, $\left.\eta_{p}{ }^{2}=0.321\right)$. The CONTEXT $\mathrm{x}$ RESPONSE interaction was marginally significant $\left(F_{(1,29)}=3.31, p=\right.$ $\left.0.059, \eta_{p}{ }^{2}=0.102\right)$. Follow-up paired t-tests confirmed a larger CRN amplitude for slow $(M=-3.58 \mu \mathrm{V}$, 
$\mathrm{SD}=3.62)$ than fast hits $(M=-2.22 \mu \mathrm{V}, \mathrm{SD}=3.53)$ in the irrelevant context $\left(t_{(29)}=3.94, p<0.001\right)$, while this effect was not significant in the relevant context (with $M=-4.02 \mu \mathrm{V}, \mathrm{SD}=3.61$, and $M=-3.75 \mu \mathrm{V}$, $\mathrm{SD}=3.27$ for slow and fast responses, respectively; $\left.t_{(29)}=1.93, p=0.063\right)$.

\section{[insert Figure 5 here]}

\section{Discussion}

In everyday-life situations PM provides a flexible and adaptive mechanism to detect the occurrence of mismatches or conflicts between goals or intentions and actions, and to trigger in turn remedial processes (Ullsperger et al., 2014b). Usually, this PM is achieved through the processing of specific internal (motorbased) states or values, and/or the use of external (feedback-based) incentives or visual stimuli provided in the proximal environment. According to the first indicator hypothesis (Bediou, Koban, Rosset, Pourtois, \& Sander, 2012; Gentsch, Ullsperger, \& Ullsperger, 2009; Stahl, 2010) and the reward prediction error account (Holroyd \& Coles, 2002), PM is a thrifty process in the sense that it uses the information available at a given moment in time (either response/motor- or feedback-based), thereby avoiding the need to perform multiple checks or monitoring across successive time epochs. When response/motor-based evidence lacks or has not accumulated properly, PM mainly operates based on the processing of external evaluative feedback (if available), usually provided in the form of a binary outcome (success/win or failure/loss). At the electrophysiological level, these two different stages of PM (internal and external) are captured by amplitude variations of the CRN/ERN and FRN components, respectively (Ullsperger et al., 2014b). In this work, we mainly sought to test the prediction that PM is flexible and accordingly, it depends upon specific contextual or environmental factors. Central to this study was the prediction that goal relevance in the sense of feedback reliability can shape PM, with effects visible at the level of the FRN component, thereby challenging the assumption that this ERP component reflects the operation of a monitoring mechanism that is exclusively based on the valence or expectedness of the feedback stimuli (Hajcak, Holroyd, Moser, \& Simons, 2005). 
To test this prediction, in two distinct ERP experiments, participants carried out a speeded Go/NoGo task with evaluative feedback informing them about the goal conduciveness of their actions (Aarts \& Pourtois, 2010; 2012; Koban et al., 2012; Vocat et al., 2008). Critically, using a standard visual cueing technique and specific instructions, we manipulated the perceived goal relevance of the feedback by varying the objective goal relevance of the feedback across successive blocks, while keeping all other features of the task unchanged: In some blocks, the feedback was informative about actual task performance, while in the other blocks it was not, and therefore conveyed evaluative information that was unrelated to the actual performance. To make sure that participants attended to the feedback stimuli equally well in the two contexts (and hence to balance the overall task relevance of the feedback; see Folk \& Remington, 2008), we added catch trials in which participants were occasionally asked to judge the emotional content of the face used as the feedback stimulus. Results for these catch trials (see Table 1) confirmed that participants attended to the feedback stimuli equally so in the two contexts. Moreover, manipulation checks confirmed that feedback was perceived as more relevant in the relevant than the irrelevant context. Critically, our new ERP results showed that this manipulation substantially influenced the amplitude of the FRN component. In Experiment 1, we found an amplitude difference between negative and positive feedback in the relevant context (Miltner et al., 1997; Nieuwenhuis et al., 2004), but not in the irrelevant one. Moreover, the amplitude of this negative component was overall larger for feedback shown in the irrelevant than the relevant context. This latter result was replicated in Experiment 2 where the probability of positive vs. negative feedback was balanced in the two contexts, confirming that reward expectancy does not solely account for amplitude variations at the level of the FRN component (but see Ferdinand et al., 2012). Moreover, following the FRN, we found a clear modulation of the parietal P3b with goal relevance, with this component being larger for relevant than irrelevant feedback. Interestingly, at the response level, we found a symmetrical outcome (compared to the P3b): Internal monitoring (and more specifically, the differentiation between fast/correct and slow/incorrect hits at the CRN level) was enhanced in the irrelevant context (where the external feedback provided was 
deemed uninformative) compared to the relevant one. Below, we discuss the implications of these new ERP results in greater detail.

\subsection{Hierarchical PM effects at the FRN level}

Collectively, the new ERP results obtained in Experiments 1 and 2 suggest that the FRN component reflects the processing of goal relevance in the sense of the reliability (and/or the impact) of the feedback stimulus for goals. In both experiments the FRN amplitude differed between the irrelevant and the relevant condition, suggesting that this contextual manipulation did shape PM rapidly following feedback onset. Moreover, only in the relevant context there was a differential FRN effect reported between positive and negative feedback, when the former was infrequent compared to the latter (Experiment 1). This valence-specific effect is compatible with the dominant reward prediction error account (Holroyd \& Coles, 2002; Proudfit, 2015) emphasizing the combined role of valence and expectedness during PM, as well as more recent ERP results emphasizing the importance of expectedness at the root of the FRN (Ferdinand et al., 2012). However, our new ERP findings significantly extend these earlier models and results by suggesting a possible hierarchy among different PM processes operating rapidly following feedback onset (at the FRN level). More specifically, goal relevance appears to be a pre-requisite for effects of valence and/or expectedness at the FRN level. Furthermore, expectedness effects seemed to be a pre-requisite for valence effects. Indeed, when the expectedness of the feedback was carefully balanced (in Experiment 2), the FRN valence effect was strongly attenuated (relative to Experiment 1 in which positive feedback stimuli were less frequent than negative feedback stimuli, and a clear valence effect was found at the FRN level in the relevant context only), and it was apparently delayed to the P3b component (see below). This suggests that amplitude variations of the FRN component might depend upon different (monitoring) processes operating concurrently, which differ from one another regarding their actual level of abstractness within a putative hierarchy of medial frontal (or lateral frontal) brain structures timely engaged during PM and, more generally, cognitive control (see Badre, 2008 for a plausible neuroanatomical model based on fMRI and patient data, suggesting a rostro-caudal organization or gradient in 
lateral prefrontal cortex). With this scenario, goal relevance would provide a superordinate level of processing during PM, with expectedness occupying an intermediate level, and valence perhaps a subordinate level. Given the limited spatial resolution of ERPs, it appears difficult to corroborate this assumption directly, however, and future (multimodal) studies are needed to assess whether amplitude variations at the FRN level during PM might be explained by multiple sources arising from the prefrontal cortex and obeying a rostro-caudal anatomical organization.

The lack of a differential FRN (valence) effect when positive and negative feedback were carefully balanced in the relevant condition (Experiment 2) is actually in agreement with many previous ERP studies (see Holroyd \& Coles, 2002; Ferdinand et al., 2012), which already suggested that reward probability is an important variable modulating the amplitude of the FRN component (i.e., reward prediction error account; Holroyd \& Coles, 2002). In addition, our new ERP results align with a recent theoretical model (see Ullsperger, Danielmeier, \& Jocham, 2014a) assuming that PM is not a fixed or rigid process, but that dynamic changes or adjustments in this process can be observed: If (reward) probability is no longer informative (see Experiment 2, relevant context), then feedback processing appears to be postponed to later stages (i.e., the P3b component, see below).

In the case of a strong imbalance between positive and negative feedback (in the relevant context of Experiment 1), not only the amplitude of the FRN component (250-300 ms post-feedback onset), but also the subsequent frontal P3a (350-450 ms post-feedback onset) was reliably influenced by this valence/expectedness effect (being larger for positive/infrequent than negative/frequent feedback). This confirmed the sensitivity of this later ERP component to the detection of positive, salient and infrequent or unexpected stimuli (Polich, 2007). No such P3a effect was evidenced when the two feedback types were equiprobable (in the irrelevant contexts and the relevant context of Experiment 2). However, in this case, the main effect of goal relevance was still significant during the FRN time course, suggesting that the P3a and FRN reflect different stages of feedback/stimulus processing during PM (see also von Borries et al., 2013). Our auxiliary ERP topographical mapping analysis also confirmed that these two successive ERP components could be dissociated from one another (see Methods section). 
It must be noted that previous psychophysiological research already provided hints about the importance of goal relevance of the feedback to explain amplitude modulations of well-known feedbacklocked ERP components (such as the FRN and P3/LPC) during PM (see also Gibbons et al., 2016; Osinsky, Walter, \& Hewig, 2014). For example, Fischer and Ullsperger (2013) previously reported a larger FRN to negative outcomes during a probabilistic reinforcement learning task when compared with favorable ones, but exclusively in a condition/context in which participants had chosen to attend to the feedback. In this earlier study, no such FRN (valence) effect was reported in a condition in which participants had chosen to avoid it. Moreover, these authors also reported a larger P3b component for unfavorable compared to favorable feedback, but this effect was equally strong in the chosen and avoided contexts. It must be noted, however, that the procedure devised by Fischer and Ullsperger (2013) is not entirely comparable with the context manipulation used in our study. The main difference is that feedback information provided in the irrelevant context of our study was completely uninformative, while feedback in the avoided condition of Fischer and Ullsperger (2013) was informative to some degree, as participants probably still could decipher what they could have won or lost if they had actually chosen to receive it (instead of avoiding it). As feedback was informative (goal-relevant) to some degree in both conditions in their study, this can therefore potentially explain why the P3b component was not statistically different between their avoided vs. chosen condition. Moreover, an asset of our experimental design was that, unlike Fischer and Ullsperger (2013), task relevance (and hence attention allocated to the feedback) was balanced between the two contexts. In another ERP study using a gambling task, Gentsch and colleagues (2013) provided evidence for sequential appraisal effects taking place at the level of the FRN and P300 components, with the former ERP deflection being related to the appraisal of goal conduciveness and the latter component to the appraisal of coping potential. Finally, Holroyd, Krigolson, Baker, Lee, and Gibson (2009) already elegantly demonstrated the sensitivity of the FRN amplitude to reward probability during PM depending on the specific (reinforcement learning) context (or trial history) used, and speculatively depending on its relevance. Their results showed that the FRN amplitude tracked reward probability, particularly in tasks where an optimal response could be learned from previous trial encounters, in contrast 
to tasks where no such learning could take place. Although Holroyd et al. (2009) did not interpret their ERP findings in terms of goal relevance either, one could nonetheless assume that this factor (i.e., the amount of information provided or conveyed by the feedback concerning the actual goal conduciveness of actions) could explain these results obtained for the FRN. However, an important difference between this earlier ERP study (or the study of Fischer \& Ullsperger, 2013) and ours pertains to the lack of any learning (or reinforcement learning) component in our study, in which a simple speeded Go/NoGo task was used throughout all blocks. Accordingly, our new ERP results align with those of Holroyd et al. (2009) and Fischer and Ullsperger (2013), but they also extend them significantly by showing that effects of goal relevance on the FRN component during PM could be evidenced in experimental contexts devoid of learning.

In the two experiments alike, the FRN amplitude reliably differed between the two contexts, being larger (i.e., more negative) in response to irrelevant than to relevant feedback. This could tentatively be explained by the fact that on average in half of the trials in the irrelevant context participants received feedback information that actually mismatched with the outcome of the preceding internal monitoring process. This type of mismatch may have led to the augmentation of the FRN component, which is known to be sensitive to prediction error. Additional ERP studies are needed, however, to confirm this interpretation.

Lastly, even though feedback processing was modulated in amplitude 250-300 ms post-feedback onset at fronto-central sites as a function of goal relevance, and this effect therefore overlapped (in space and time) with the time-course of the FRN, this does not imply that it necessarily reflected a canonical FRN component, or a modulation of this PM component exclusively. The use of multiple and different facial expressions as feedback stimuli on task performance in our experiments (as opposed to simpler, binary symbolic feedback stimuli, as used repeatedly in previous ERP studies), might potentially explain the specific topography and morphology of the FRN effect reported in this study. 
4.2. Motivational salience or updating of feedback information reflected in the amplitude of the P3b component

Another important contribution of our ERP study is to show systematic amplitude variations of the subsequent parietal P3b as a function of goal relevance, albeit of a different nature than the preceding FRN (and P3a). Across the two experiments, the P3b was substantially larger for relevant than irrelevant feedback. Consistent with previous ERP findings (see von Borries et al., 2013), this long-latency effect could reflect an enhanced attention allocation to significant or salient (emotional) information available in the environment, or even the updating of relevant stimulus information (Donchin \& Coles, 1998; Polich, 2007). Previous ERP studies already confirmed the link between the amplitude of the P3b component and the processing of stimuli meant to update or alter existing/prior knowledge or information about them (Hajcak et al., 2006; 2007). Translated to our new ERP results, external feedback on task performance provided in the relevant context triggered an updating or enhanced (motivational) processing, relative to the same feedback provided in the irrelevant context and lacking thereby motivational significance. Noteworthy, this P3b amplitude effect (or the preceding FRN or P3a effects) cannot be explained by asymmetrical or uncontrolled attentional or task relevance effects between the two contexts since in both cases the feedback was always task-relevant (as ensured by the use of specific catch trials). Additionally, in Experiment 2, we found that negative feedback provided in the relevant context led to a larger P3b component than positive feedback provided in the same context, suggesting some flexibility in the amount of stimulus updating achieved in this condition. Presumably, negative feedback conveying a punishmentrelated meaning (or informing about self-efficacy failure) had a larger impact on stimulus updating than positive feedback in the relevant context in this experiment (see also Fischer \& Ullsperger, 2013). Relatedly, at the behavioral level, we also found that participants judged the feedback to be more reliable in the relevant condition in Experiment 2 than Experiment 1. Alternatively, it is also conceivable that in Experiment 2, in which feedback expectancy was controlled for, valence-based PM did not operate at the FRN level, but was postponed to the P3b. Importantly, however, in Experiment 2, goal relevance did 
modulate the amplitude of the FRN component. As such, our ERP results emphasize the flexibility of these PM brain processes (based on external feedback processing), and they contradict earlier theoretical accounts that posit context-independent monitoring of either valence or expectedness (or both) at the FRN or P3 level (Hajcak et al., 2005; 2006). Our new ERP results clearly suggest that when feedback expectancy could not be used by the participants to assign 'online' a different value to either positive or negative feedback on task performance (Experiment 2), then the (normal) valence effect at the FRN level occurred later in time following feedback onset, namely at the P3b level. Importantly, our new findings show for the first time the strong dependence of these flexible neurophysiological effects reflecting PM to goal relevance, conceived here as feedback reliability.

\subsection{Flexible external and internal PM effects}

According to dominant models (Holroyd \& Coles, 2002; Ullsperger et al., 2014a), PM is a fairly dynamic and flexible process. It seeks to minimize redundancy and utilises the information available at a given moment in time (either response- or feedback-based) to guide behavior. This enables rapid adjustments and an efficient updating of actions' value depending on the availability of contextual cues or incentives available in the environment. To verify this assumption, we tested whether our manipulation of goal relevance, by means of contextual cues and written instructions, also influenced response-locked ERPs (in addition to the feedback-locked ERP data discussed above), with a focus on the CRN component (Coles, Scheffers, \& Holroyd, 2001; Roger, Bénar, Vidal, Hasbroucq, \& Burle, 2010). In line with our hypotheses, we found in the two experiments alike that in the irrelevant context (where the feedback provided on task performance was deemed uninformative) internal monitoring was transiently enhanced, as expressed by a larger CRN differentiation between slow/incorrect and fast/correct hits in this context compared to the relevant one. These complementing results are important because they lend support to the notion of flexible and adaptive PM effects in humans (Ullsperger et al., 2014a), with an apparent shift of the monitoring process (from external to internal cues) when externally provided feedback on task performance is no longer reliable (i.e., in the irrelevant context). Notably, the observation that goal 
relevance can shape not only the way evaluative feedback (external cues), but also motor responses (internal cues) are timely processed during PM is another important contribution of our study.

\subsection{Conclusions}

The present study shows that PM brain processes (captured by the FRN, P3, and CRN components) are flexible and reliably shaped by contextual effects related to goal relevance. In the present case, we operationalized goal relevance as the reliability of specific incentives (i.e., evaluative feedback) informing participants about the goal conduciveness of their actions, and we made it variable across successive blocks using a within-subjects design while controlling for all other task dimensions. When participants were cued that feedback provided on task performance was disconnected from their behavior/decision, the FRN component no longer distinguished rewards from punishments (Experiment 1), as if the normal PM processes were inactive or transiently suppressed. This suggests that goal relevance influences early stages of PM based on feedback. Importantly, in this condition (irrelevant context), an enhanced internal monitoring (at the CRN level) was found. Moreover, our results confirm that feedback expectancy is an important variable accounting for amplitude modulations occurring at the FRN level during PM. When reward and punishment probability were matched (as in the relevant context of Experiment 2), the processing of these two opposite outcomes was delayed and took place at the P3b level. As such, these new findings largely accord with dominant PM models in the literature (Holroyd \& Coles, 2002; Proudfit, 2015; Ullsperger et al., 2014a), while they also open new avenues for a better conceptualization and integration of goal relevance into these models. 
References

Aarts, K., \& Pourtois, G. (2010). Anxiety not only increases, but also alters early error-monitoring functions. Cognitive, Affective, \& Behavioral Neuroscience, 10, 479-492, doi:10.3758/CABN.10.4.479.

Aarts, K., \& Pourtois, G. (2012). Anxiety disrupts the evaluative component of performance monitoring: An ERP study. Neuropsychologia,50, 1286-1296, doi:10.1016/j.neuropsychologia. 2012.02.012.

Atkinson, J. W., \& Feather, N. T. (1966). A theory of achievement motivation. New York: Wiley.

Badre, D. (2008). Cognitive control, hierarchy, and the rostro-caudal organization of the frontal lobes. Trends in Cognitive Sciences, 12, 193-200, doi:10.1016/j.tics.2008.02.004.

Bediou, B., Koban, L., Rosset, S., Pourtois, G., \& Sander, D. (2012). Delayed monitoring of accuracy errors compared to commission errors in ACC. Neurolmage, 60, 1925-1936, doi:10.1016/j.neuroimage.2012.02.010.

Bismark, A. W., Hajcak, G., Whitworth, N. M., \& Allen, J. J. (2013). The role of outcome expectations in the generation of the feedback-related negativity. Psychophysiology, 50, 125-133, doi: 10.1111/j.1469-8986.2012.01490.x.

Coles, M. G., Scheffers, M. K., \& Holroyd, C. B. (2001). Why is there an ERN/Ne on correct trials? Response representations, stimulus-related components, and the theory of error-processing. Biological Psychology, 56, 173-189, doi:10.1016/S0301-0511(01)00076-X.

Defares, P. B., van der Ploeg, H. M., \& Spielberger, C. D. (1979). Zelf-beoordelings vragenlijst. Lisse: Swets \& Zeitlinger.

Dhar, M., \& Pourtois, G. (2011). Early error detection is generic, but subsequent adaption to errors is not: evidence from ERPs. Neuropsychologia, 49, 1236-1245, doi: 10.1016/j.neuropsychologia.2011.01.006. 
Dhar, M., Wiersema, J. R., \& Pourtois, G. (2011). Cascade of neural events leading from error commission to subsequent awareness revealed using EEG source imaging. PLoS One, 6, e19578, doi: 10.1371/journal.pone.0019578.

Donchin, E., \& Coles, M. G. (1998). Context updating and the P300. Behavioral and Brain Sciences, 21, 152-154, doi: http://dx.doi.org/10.1017/S0140525X98230950.

Eitam, B., \& Higgins, E. T. (2010). Motivation in mental accessibility: Relevance of a representation (ROAR) as a new framework. Social and Personality Psychology Compass, 4, 951-967, doi: 10.1111/j.1751-9004.2010.00309.x.

Ferdinand, N. K., Mecklinger, A., Kray, J., \& Gehring, W. J. (2012). The processing of unexpected positive response outcomes in the mediofrontal cortex. The Journal of Neuroscience, 32, 12087-12092, doi: 10.1523/JNEUROSCI.1410-12.2012.

Fischer, A. G., \& Ullsperger, M. (2013). Real and fictive outcomes are processed differently but converge on a common adaptive mechanism. Neuron, 79, 1243-1255, doi:10.1016/j.neuron.2013.07.006.

Folk, C. L., \& Remington, R. W. (2008). Bottom-up priming of top-down attentional control settings. Visual Cognition, 16, 215-231, doi:10.1080/13506280701458804.

Frijda, N. H. (1986). The emotions. Cambridge University Press.

Gehring, W. J., \& Willoughby, A. R. (2002). The medial frontal cortex and the rapid processing of monetary gains and losses. Science, 295, 2279-2282, doi: 10.1126/science.1066893.

Gentsch, A., Ullsperger, P., \& Ullsperger, M. (2009). Dissociable medial frontal negativities from a common monitoring system for self-and externally caused failure of goal achievement. NeuroImage, 47, 2023-2030, doi:10.1016/j.neuroimage.2009.05.064.

Gentsch, K., Grandjean, D., \& Scherer, K. R. (2013). Temporal dynamics of event-related potentials related to goal conduciveness and power appraisals. Psychophysiology, 50, 1010-1022, doi: 10.1111/psyp.12079. 
Gibbons, H., Schnuerch, R., \& Stahl, J. (2016). From positivity to negativity bias: Ambiguity affects the neurophysiological signatures of feedback processing. Journal of Cognitive Neuroscience, 14, 1-16, doi: 10.1162/jocn_a_00921.

Gratton, G., Coles, M. G., \& Donchin, E. (1983). A new method for off-line removal of ocular artifact. Electroencephalography and Clinical Neurophysiology, 55, 468-484, doi:10.1016/00134694(83)90135-9.

Hajcak, G., Holroyd, C. B., Moser, J. S., \& Simons, R. F. (2005). Brain potentials associated with expected and unexpected good and bad outcomes. Psychophysiology, 42, 161-170, doi: 10.1111/j.14698986.2005.00278.x.

Hajcak, G., Moser, J. S., Holroyd, C. B., \& Simons, R. F. (2006). The feedback-related negativity reflects the binary evaluation of good versus bad outcomes. Biological Psychology, 71, 148-154, doi:10.1016/j.biopsycho.2005.04.001.

Hajcak, G., Moser, J. S., Holroyd, C. B., \& Simons, R. F. (2007). It's worse than you thought: The feedback negativity and violations of reward prediction in gambling tasks. Psychophysiology, 44, 905912, doi: 10.1111/j.1469-8986.2007.00567.x.

Holroyd, C. B., \& Coles, M. G. (2002). The neural basis of human error processing: reinforcement learning, dopamine, and the error-related negativity. Psychological Review, 109, 679-709, doi: http://dx.doi.org/10.1037/0033-295X.109.4.679.

Holroyd, C. B., Krigolson, O. E., Baker, R., Lee, S., \& Gibson, J. (2009). When is an error not a prediction error? An electrophysiological investigation. Cognitive, Affective, \& Behavioral Neuroscience, 9, 59-70, doi: 10.3758/CABN.9.1.59.

Holroyd, C. B., Pakzad-Vaezi, K. L., \& Krigolson, O. E. (2008). The feedback correct-related positivity: Sensitivity of the event-related brain potential to unexpected positive feedback. Psychophysiology, 45, 688-697, doi: 10.1111/j.1469-8986.2008.00668.x.

Jeffreys, D. A., \& Tukmachi, E. S. A. (1992). The vertex-positive scalp potential evoked by faces and by objects. Experimental Brain Research, 91, 340-350, doi: 10.1007/BF00231668. 
Jeffreys, D. A. (1993). The influence of stimulus orientation on the vertex positive scalp potential evoked by faces. Experimental Brain Research, 96, 163-172, doi: 10.1007/BF00230449.

Kahneman, D., \& Tversky, A. (1984). Choices, values, and frames. American Psychologist, 39, 341-350, doi: http://dx.doi.org/10.1037/0003-066X.39.4.341.

Keil, A., Debener, S., Gratton, G., Junghöfer, M., Kappenman, E. S., Luck, S. J., Luu, P., Miller, G. A., \& Yee, C. M. (2014). Committee report: Publication guidelines and recommendations for studies using electroencephalography and magnetoencephalography. Psychophysiology, 51, 1-21, doi: 10.1111/psyp.12147.

Koban, L., Pourtois, G., Vocat, R., \& Vuilleumier, P. (2010). When your errors make me lose or win: Event-related potentials to observed errors of cooperators and competitors. Social Neuroscience,5, 360-374, doi: 10.1080/17470911003651547.

Koban, L., Pourtois, G., Bediou, B., \& Vuilleumier, P. (2012). Effects of social context and predictive relevance on action outcome monitoring. Cognitive, Affective, \& Behavioral Neuroscience, 12, 460-478, doi: 10.3758/s13415-012-0091-0.

Lundqvist, D., Flykt, A., \& Öhman, A. (1998). The Karolinska Directed Emotional Faces $K D E F, \mathrm{CD}$ ROM from Department of Clinical Neuroscience, Psychology section, Karolinska Institutet, Stockholm, Sweden.

Miltner, W. H., Braun, C. H., \& Coles, M. G. (1997). Event-related brain potentials following incorrect feedback in a time-estimation task: Evidence for a "generic" neural system for error detection. Journal of Cognitive Neuroscience, 9, 788-798, doi:10.1162/jocn.1997.9.6.788.

Moors, A. (2007). Can cognitive methods be used to study the unique aspect of emotion: An appraisal theorist's answer. Cognition and Emotion, 21, 1238-1269, doi:10.1080/02699930701438061.

Murray, M. M., Brunet, D., \& Michel, C. M. (2008). Topographic ERP analyses: a step-by-step tutorial review. Brain Topography, 20, 249-264, doi: 10.1007/s10548-008-0054-5. 
Nieuwenhuis, S., Holroyd, C. B., Mol, N., \& Coles, M. G. (2004). Reinforcement-related brain potentials from medial frontal cortex: origins and functional significance. Neuroscience \& Biobehavioral Reviews, 28, 441-448, doi:10.1016/j.neubiorev.2004.05.003.

Osinsky, R., Walter, H., \& Hewig, J. (2014). What is and what could have been: An ERP study on counterfactual comparisons. Psychophysiology, 51, 773-781, doi: 10.1111/psyp.12221.

Pfabigan, D. M., Alexopoulos, J., Bauer, H., Lamm, C., \& Sailer, U. (2011). All about the money - external performance monitoring is affected by monetary, but not by socially conveyed feedback cues in more antisocial individuals. Frontiers in Human Neuroscience, 5, doi: 10.3389/fnhum.2011.00100.

Pfabigan, D. M., Zeiler, M., Lamm, C., \& Sailer, U. (2014). Blocked versus randomized presentation modes differentially modulate feedback-related negativity and P3b amplitudes. Clinical Neurophysiology, 125, 715-726, doi:10.1016/j.clinph.2013.09.029.

Polich, J. (2007). Updating P300: an integrative theory of P3a and P3b. Clinical Neurophysiology, 118, 2128-2148, doi:10.1016/j.clinph.2007.04.019.

Pourtois, G. (2011). Early error detection predicted by reduced pre-response control process: an ERP topographic mapping study. Brain Topography, 23, 403-422, doi: 10.1007/s10548-010-0159-5.

Pourtois, G., De Pretto, M., Hauert, C. A., \& Vuilleumier, P. (2006). Time course of brain activity during change blindness and change awareness: performance is predicted by neural events before change onset. Journal of Cognitive Neuroscience, 18, 2108-2129, doi:10.1162/jocn.2006.18.12.2108.

Pourtois, G., Delplanque, S., Michel, C., \& Vuilleumier, P. (2008). Beyond conventional eventrelated brain potential (ERP): exploring the time-course of visual emotion processing using topographic and principal component analyses. Brain Topography, 20, 265-277, doi: 10.1007/s10548-008-0053-6.

Proudfit, G. H. (2015). The reward positivity: From basic research on reward to a biomarker for depression. Psychophysiology, 52, 449-459, doi: 10.1111/psyp.12370.

Roger, C., Bénar, C. G., Vidal, F., Hasbroucq, T., \& Burle, B. (2010). Rostral Cingulate Zone and correct response monitoring: ICA and source localization evidences for the unicity of correct-and errornegativities. NeuroImage, 51, 391-403, doi:10.1016/j.neuroimage.2010.02.005. 
Roseman, I. J., \& Smith, C. A. (2001). Appraisal theory: Overview, assumptions, varieties, controversies. In: K. R. Scherer, A. Schorr, \& T. Johnstone (Eds.), Appraisal Processes in Emotion: Theory, Methods, Research. Oxford University Press, New York and Oxford, pp. 3-19.

Rotter, J. B. (1966). Generalized expectancies for internal versus external control of reinforcement. Psychological Monographs, 80, 1-28, doi: http://dx.doi.org/10.1037/h0092976.

Sambrook, T. D., \& Goslin, J. (2015). A neural reward prediction error revealed by a metaanalysis of ERPs using great grand averages. Psychological Bulletin, 141, 213-235, doi: $10.1037 /$ bul0000006.

Stahl, J. (2010). Error detection and the use of internal and external error indicators: An investigation of the first-indicator hypothesis. International Journal of Psychophysiology, 77, 43-52, doi:10.1016/j.ijpsycho.2010.04.005.

Ullsperger, M., Danielmeier, C., \& Jocham, G. (2014a). Neurophysiology of performance monitoring and adaptive behavior. Physiological Reviews, 94, 35-79, doi: 10.1152/physrev.00041.2012.

Ullsperger, M., Fischer, A. G., Nigbur, R., \& Endrass, T. (2014b). Neural mechanisms and temporal dynamics of performance monitoring. Trends in Cognitive Sciences, 18, 259-267, doi:10.1016/j.tics.2014.02.009.

Vocat, R., Pourtois, G., \& Vuilleumier, P. (2008). Unavoidable errors: a spatio-temporal analysis of time-course and neural sources of evoked potentials associated with error processing in a speeded task. Neuropsychologia, 46, 2545-2555, doi:10.1016/j.neuropsychologia.2008.04.006.

von Borries, A. K. L., Verkes, R. J., Bulten, B. H., Cools, R., \& de Bruijn, E. R. A. (2013). Feedback-related negativity codes outcome valence, but not outcome expectancy, during reversal learning. Cognitive, Affective, \& Behavioral Neuroscience, 13, 737-746, doi: 10.3758/s13415-013-0150-1.

Walsh, M. M., \& Anderson, J. R. (2012). Learning from experience: event-related potential correlates of reward processing, neural adaptation, and behavioral choice. Neuroscience \& Biobehavioral Reviews, 36, 1870-1884, doi:10.1016/j.neubiorev.2012.05.008. 


\section{Author Note}

University position of WW is supported by funding from the Jagiellonian University within the 'Society Environment - Technology (SET)' project (S/FS0/0117.30). The project is co-financed by the European Union. Her staying at Ghent University is funded by the Mobility Plus III grant (1096/MOB/2013/0), financed by the Polish Ministry of Science and Higher Education.

GP is supported by the Belgian Science Policy, Interuniversity Attraction Poles program (P7/11) and by a Concerted Research Action Grant from Ghent University. GP and AM are also supported by a research grant from the Research Foundation Flanders (FWO grant G024716N).

Conflict of interest: none declared.

Correspondence should be sent to: Wioleta Walentowska, Psychophysiology Laboratory, Jagiellonian University, Ingardena 6, 30-060 Kraków, Poland. 


\begin{tabular}{|c|c|c|c|c|c|c|}
\hline & & \multicolumn{3}{|l|}{ Accuracy } & \multirow[t]{2}{*}{ Feedback } & \multirow{2}{*}{$\begin{array}{c}\text { Speed of reactions } \\
\text { Fast hits / } \\
\text { Slow hits / } \\
\text { False alarms [ms] }\end{array}$} \\
\hline & & $\begin{array}{l}\text { Fast hits / } \\
\text { Slow hits }\end{array}$ & $\begin{array}{c}\text { False alarms / } \\
\text { Correct inhibitions / } \\
\text { Omissions }\end{array}$ & $\begin{array}{l}\text { Catch trials } \\
{[\% \text { correct }]}\end{array}$ & & \\
\hline \multirow[t]{2}{*}{ Experiment 1} & Relevant & $\begin{array}{c}40.16(2.72) \\
(12-64) / \\
79.19(2.62) \\
(56-108)\end{array}$ & $\begin{array}{c}16.23(2.04) / \\
31.2(2.04) / \\
0.81(0.27)\end{array}$ & $93.51(1.23)$ & $\begin{array}{c}40.16(2.72) \\
(12-64) / \\
79.19(2.62) \\
(56-108)\end{array}$ & $\begin{array}{c}223.53(5.03) / \\
323.17(8.39) / \\
247.65(7.45)\end{array}$ \\
\hline & Irrelevant & $\begin{array}{c}35.06(2.94) \\
(13-64) / \\
83.63(2.82) \\
(55-109) \\
\end{array}$ & $\begin{array}{c}14.01(2.04) / \\
34.45(2.04) / \\
1.21(0.48)\end{array}$ & $92.61(1.13)$ & $\begin{array}{c}59.44(1.02) \\
(48-71) / \\
59.15(1.01) \\
(47-69) \\
\end{array}$ & $\begin{array}{c}232.47(5.13) / \\
337.54(10.06) / \\
295.13(17.91)\end{array}$ \\
\hline \multirow[t]{2}{*}{ Experiment 2} & Relevant & $\begin{array}{c}56.56(3.55) \\
(22-100) / \\
62.86(3.51) \\
(20-98) \\
\end{array}$ & $\begin{array}{c}13.93(1.28) / \\
34.06(1.28) / \\
0.56(0.18)\end{array}$ & $92.69(0.97)$ & $\begin{array}{c}56.56(3.55) \\
(22-100) / \\
62.86(3.51) \\
(20-98) \\
\end{array}$ & $\begin{array}{c}246.81(5.28) / \\
336.61(6.72) / \\
256.11(7.43)\end{array}$ \\
\hline & Irrelevant & $\begin{array}{c}51.86(3.51) \\
(20-102) / \\
66.67(3.66) \\
(18-100)\end{array}$ & $\begin{array}{c}11.96(1.26) / \\
36.01(1.25) / \\
1.26(0.37)\end{array}$ & $92.65(0.96)$ & 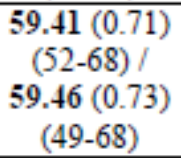 & $\begin{array}{l}283.36(7.32) / \\
369.73(7.41) / \\
302.76(18.69)\end{array}$ \\
\hline
\end{tabular}

Table 1. Behavioral results. Mean number (or percentage) and speed (with the corresponding standard error of the mean in parenthesis) provided separately for the different conditions and the two experiments. Note that average numbers of Go trials (Fast hits/Slow hits) and feedback (Positive following Fast hits, Negative following Slow hits in the relevant context, and Positive/Negative randomly following Fast/Slow hits in the irrelevant context) are presented in a bold font, and are followed by the corresponding range (minimal-maximal number of trials). At the electrophysiological level, only these trials were analyzed. 
A)

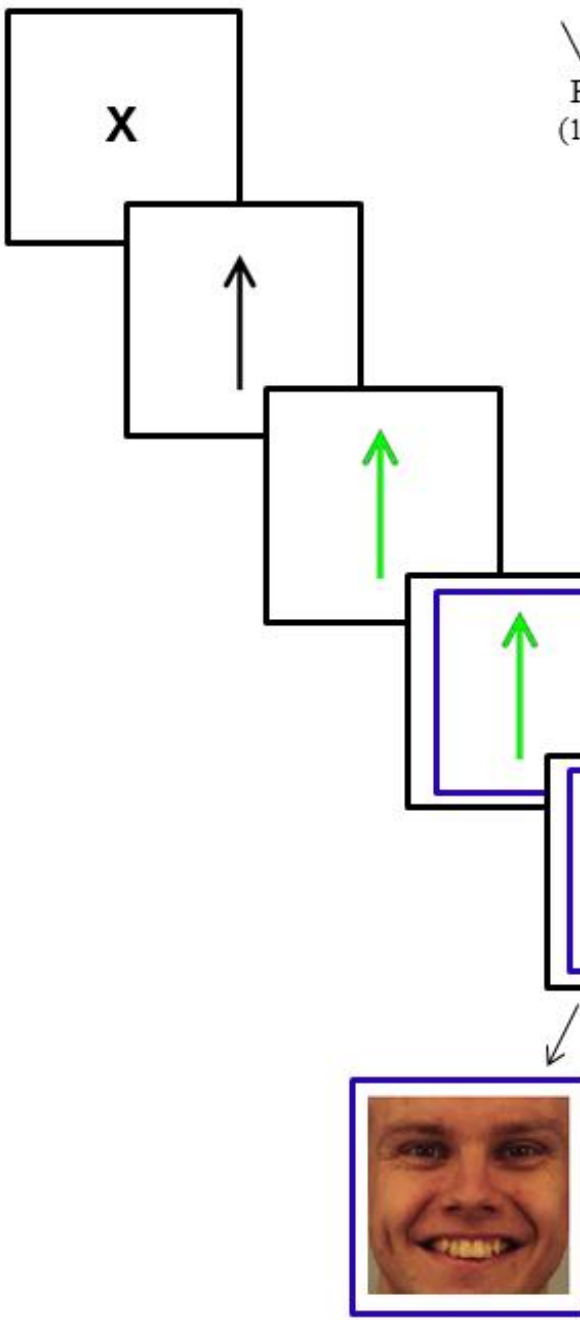

positive feedback following fast hits $\backslash$

Fixation

(1000 ms)

$\checkmark$

(1000-2000 ms)

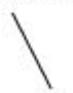

Target

(max. $1000 \mathrm{~ms})$

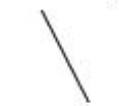

Colored frame marking

Response onset (1000 ms)

Feedback (and colored frame presented until its offset; $1000 \mathrm{~ms}$ ) negative feedback following slow hits
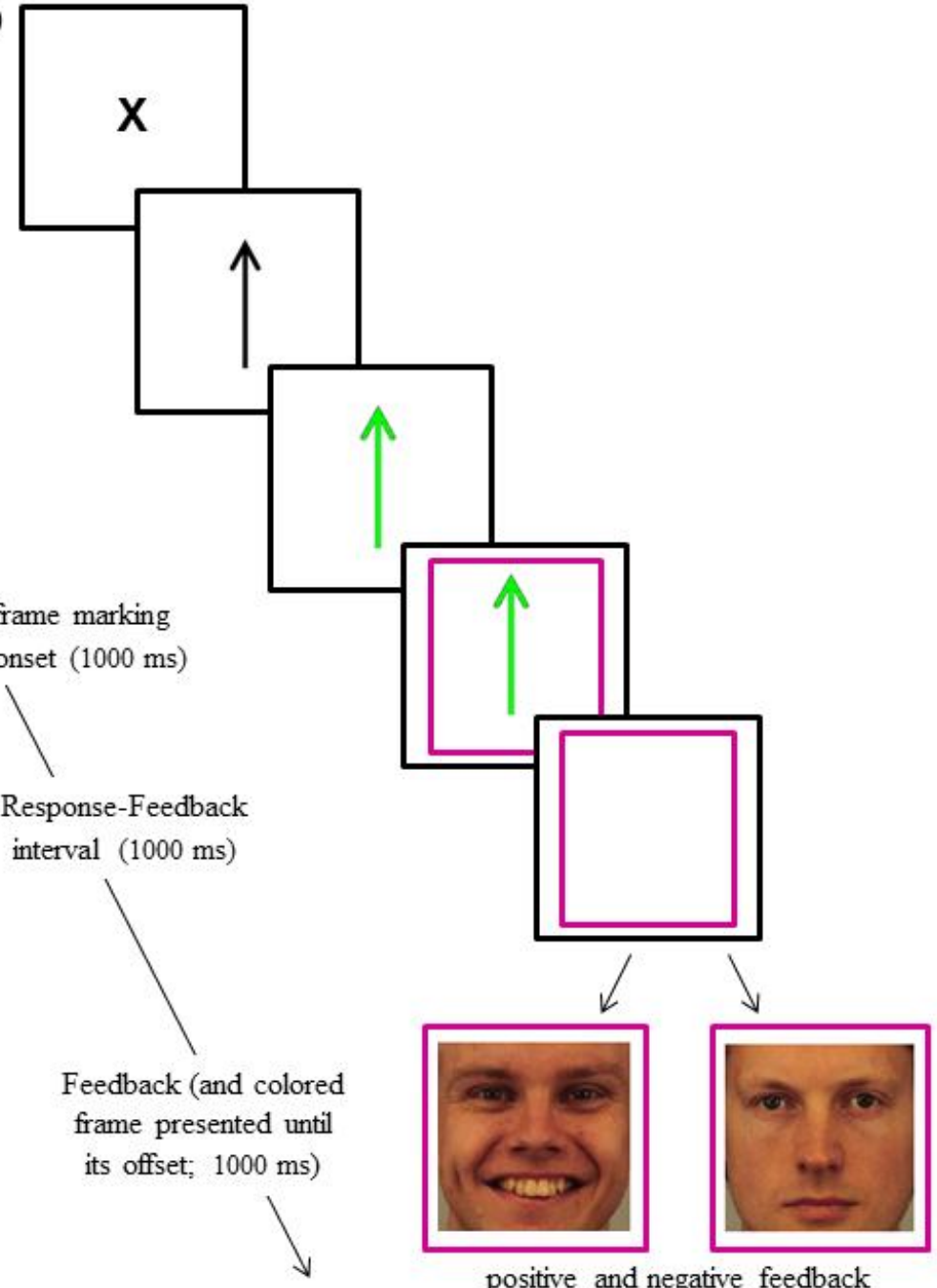

B)

positive and negative feedback randomly following fast and slow hits

Figure 1. Experimental procedure used in Experiments 1 and 2 (here illustrated for Go trials, followed by hits). (A) At response onset (speeded Go/NoGo decision; see Methods), a specific colored frame appeared around the target, signaling the response-feedback relation (relevant context), or (B) another color appeared in different blocks to signal the lack thereof (irrelevant context). This association between the color of the frame and goal relevance was alternated across participants. The frame stayed on screen until feedback offset. In the relevant context, fast hits were always followed by a positive feedback, while slow hits were always followed by a negative feedback. In contrast, in the irrelevant context, an equal amount of positive and negative feedback was presented randomly regardless of the accuracy and speed of the preceding action. 


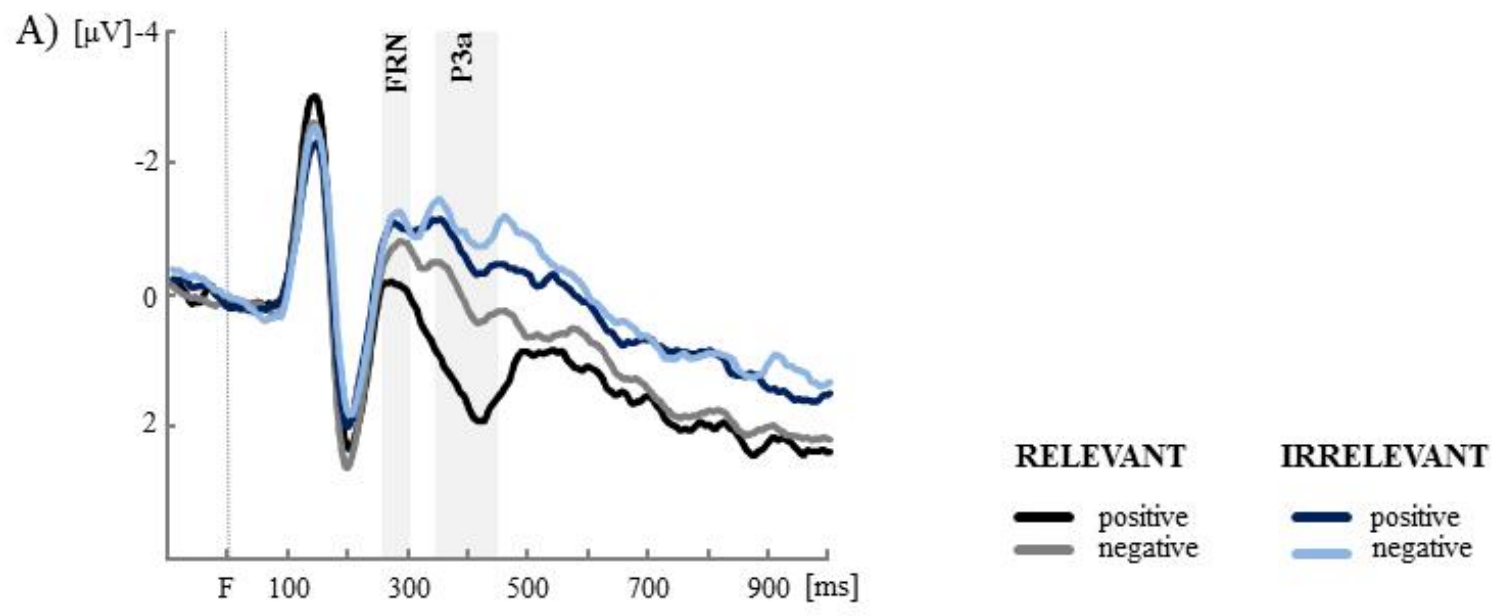

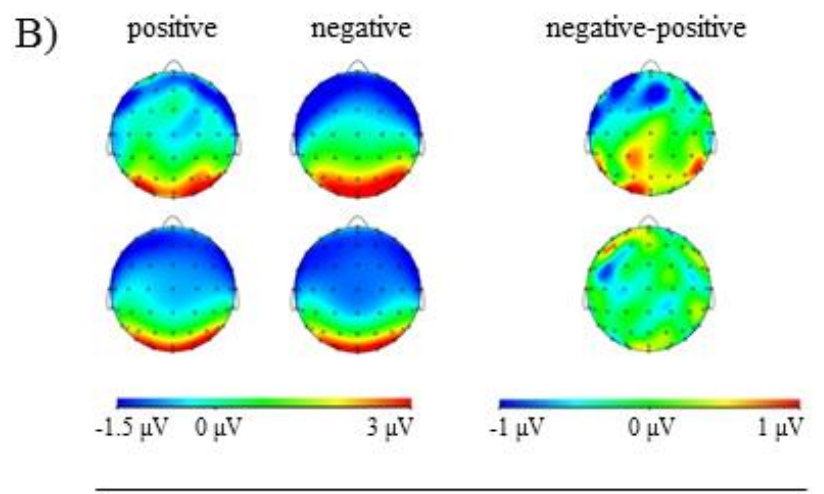

FRN

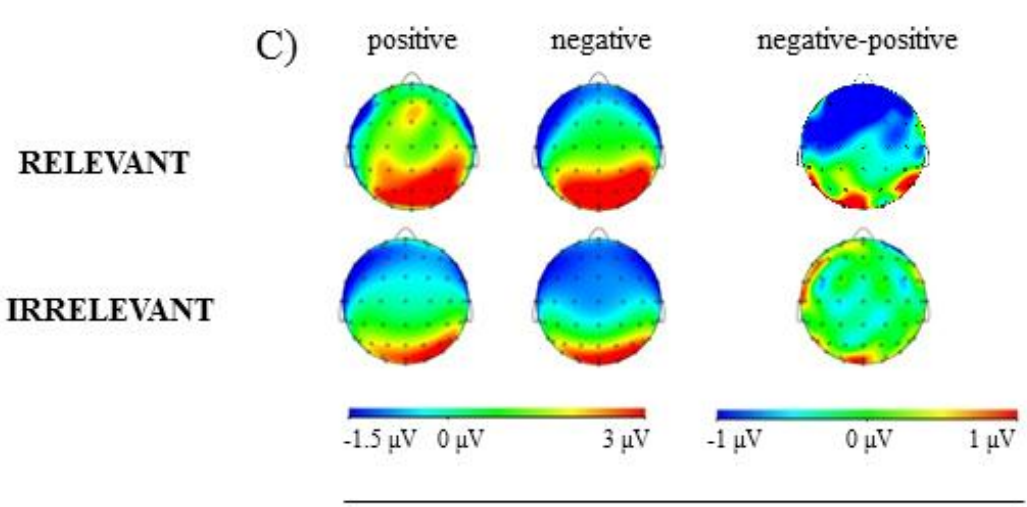

P3a

Figure 2. (A) Feedback-locked grand average ERP waveforms (Fz and FCz collapsed) for Experiment 1. The FRN (250-300 ms post-feedback onset) and the subsequent P3a (350-450 ms) had differential amplitudes for positive and negative feedback, in the relevant context only. The corresponding topographical scalp maps (horizontal view) are presented for (B) the FRN and (C) the P3a. 
A)
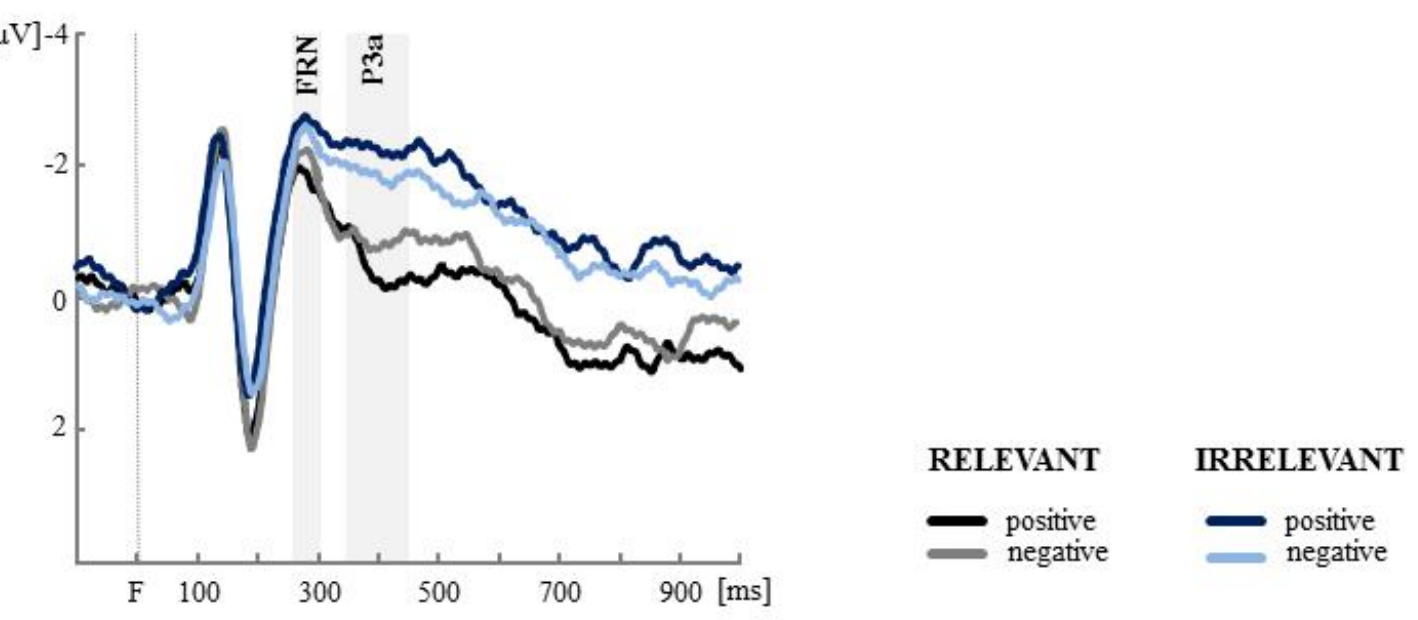

B)
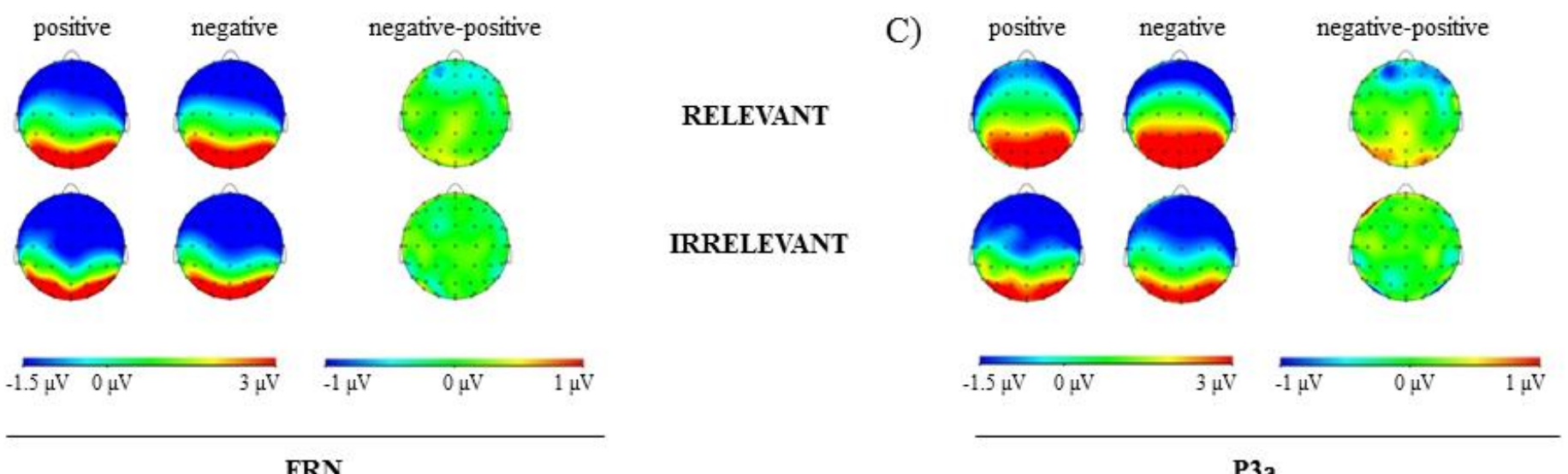

P3a

Figure 3. (A) Feedback-locked grand average ERP waveforms (Fz and FCz collapsed) for Experiment 2. The FRN (250-300 ms post-feedback onset) and the subsequent P3a (350-450 ms) had differential amplitudes for relevant than irrelevant feedback. The corresponding topographical scalp maps (horizontal view) are presented for (B) the FRN and (C) the P3a. 

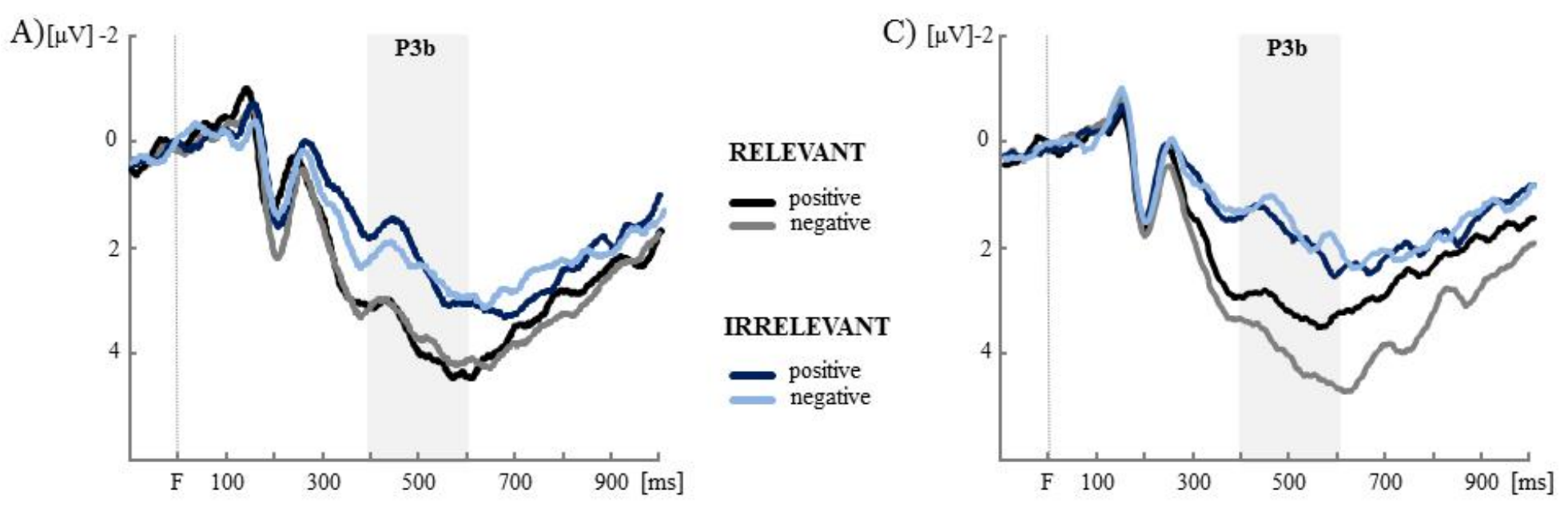

B)
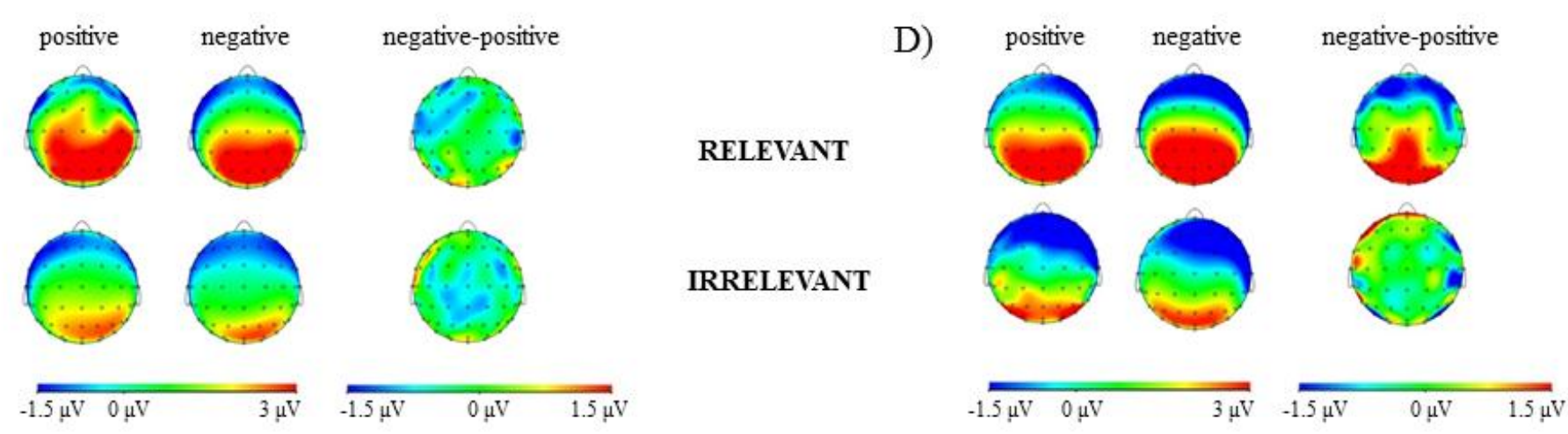

Figure 4. (A) Feedback-locked grand average ERP waveforms ( $\mathrm{CPz}, \mathrm{Pz}, \mathrm{P} 1$, and $\mathrm{P} 2$ collapsed) for Experiment 1. The P3b component (400-600 ms post-feedback onset) was larger for relevant than irrelevant feedback. (B) The corresponding topographical scalp maps (horizontal view) are presented. (C) Feedback-locked grand average ERP waveforms (CPz, Pz, P1, and P2 collapsed) for Experiment 2. The P3b component (400-600 ms post-feedback onset) was larger for relevant than irrelevant feedback. Moreover, in the relevant context only, the P3b has a larger amplitude for negative than positive feedback.

(D) The corresponding topographical scalp maps (horizontal view) are presented. 


\section{Experiment 1}

A)

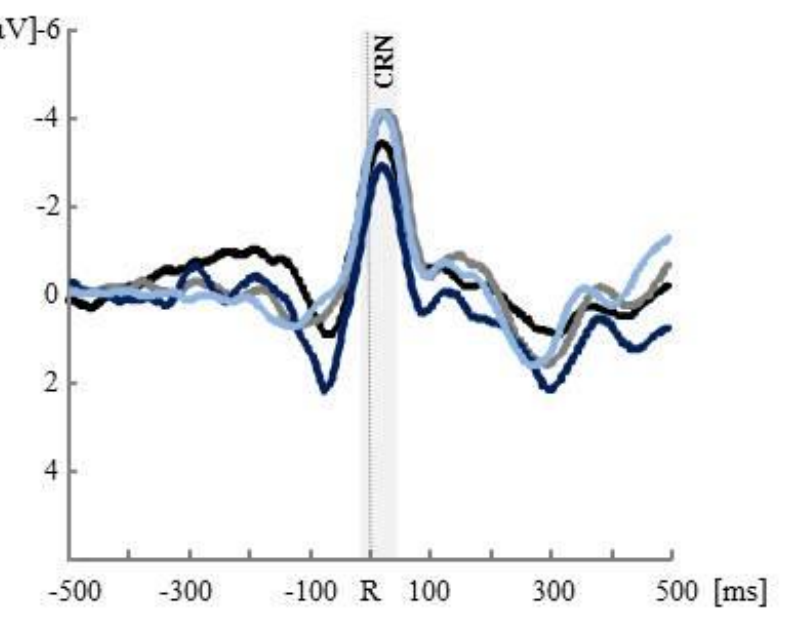

B)

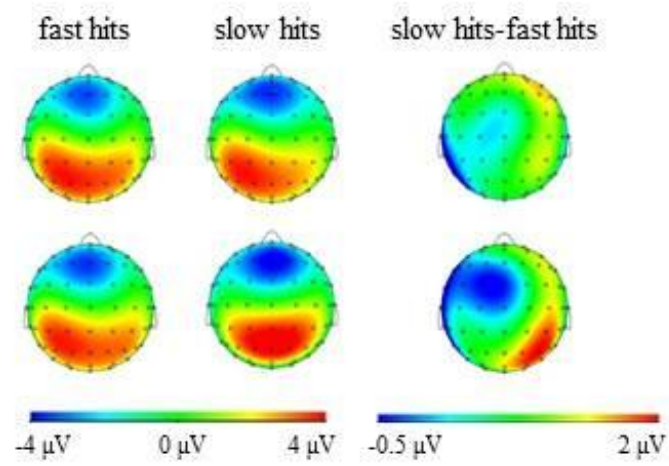

Experiment 2

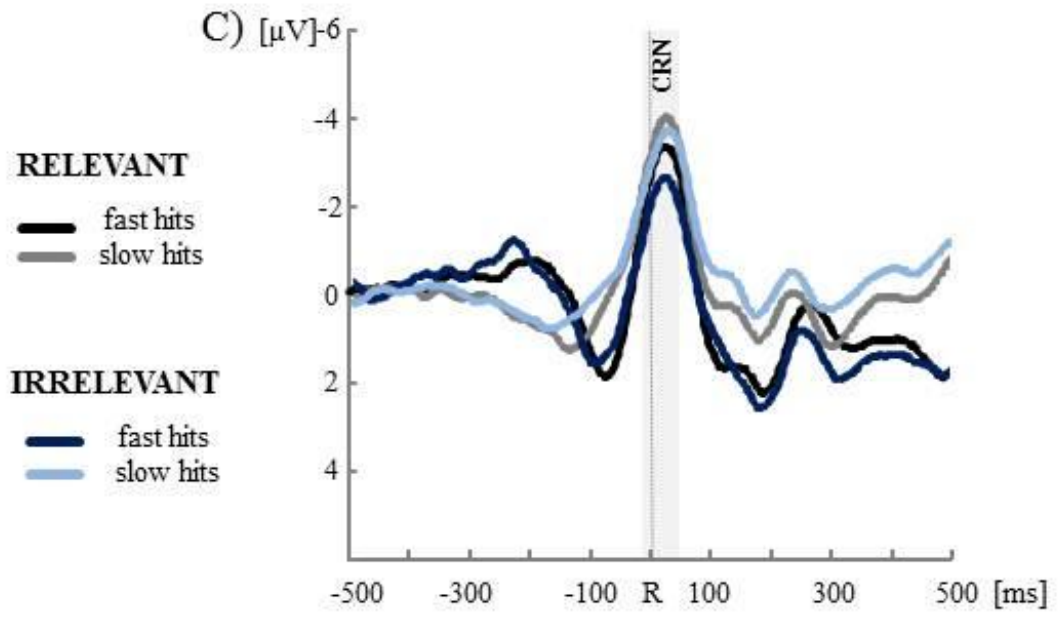

RELEVANT

D)

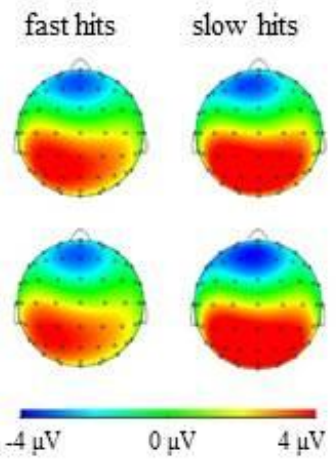

slow hits-fast hits

IRRELEVANT

Figure 5. (A) Response-locked grand average ERP waveforms (Fz and $\mathrm{FCz}$ collapsed) for Experiment 1. The CRN component (-10/+30 ms around response onset) was larger for slow hits than fast hits, in the irrelevant context only. (B) The corresponding topographical scalp maps (horizontal view) are presented. (C) Response-locked grand average ERP waveforms (Fz and FCz collapsed) for Experiment 2. The CRN component (-10/+30 ms around response onset) was larger for slow hits than fast hits, in the irrelevant context only. (D) The corresponding topographical scalp maps (horizontal view) are presented. 